\title{
Direct Visualization of Chiral Amplification of Chiral Aggregation Induced Emission Molecules in Nematic Liquid Crystals
}

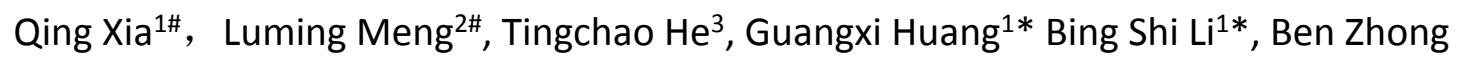
Tang ${ }^{4 *}$

${ }^{1}$ Key Laboratory of New Lithium-Ion Battery and Mesoporous Material, College of Chemistry and Environmental Engineering, Shenzhen University, 1066 Xueyuan Avenue, Nanshan, Shenzhen 518055, China.

${ }^{2}$ MOE Key Laboratory of Laser Life Science \& Guangdong Provincial Key Laboratory of Laser Life Science, College of Biophotonics, South China Normal University, Guangzhou 510631

${ }^{3}$ College of Physics and Optoelectronic Engineering, Shenzhen University, Shenzhen 518060, China.

${ }^{4}$ Department of Chemistry, Hong Kong Branch of Chinese National Engineering Research Center for Tissue Restoration and Reconstruction, The Hong Kong University of Science and Technology, Clear Water Bay, Kowloon, Hong Kong, China

Corresponding authors: Dr. Guangxi Huang *E-mail: huanggx@iccas.ac.cn Prof. Bing Shi Li *E-mail: phbingsl@szu.edu.cn_Prof. B. Z. Tang*E-mail: tangbenz@ust.hk

Miss Qing Xia ${ }^{1 \#}$ and Dr. Luming Meng ${ }^{2 \#}$ contributed equally in this paper. 


\section{Table of contents}

\section{Materials and methods}

Scheme S1-S3 Synthesis of $p$-TPE-NC, $m$-TPE-NC, and $m$-TPE-OC.

\section{Theoretical calculation}

\section{References}

Figure S1 UV and CD spectra of $p$-TPE-NC in THF.

Figure S2 DSC curves of 5CB doped with $p$-TPE-NC at the ratios of $0.1-5$ wt.\%.

Figure S3 Plots of the concentration effect of $p$-TPE-NC on CD and CPL intensity in $5 \mathrm{CB}$.

Figure S4 Fluorescence spectra of 5CB doped with $p$-TPE-NC, $p$-TPE-OC, $m$-TPE$\mathrm{NC}$ and $m$-TPE-OC.

Figure S5 DSC curves of 5CB doped with $p$-TPE-OC, $m$-TPE-OC and $m$-TPE-NC.

Figure S6 UV, CD and CPL spectra of 5CB doped with $p$-TPE-OC, $m$-TPE-NC and $m$-TPE-OC.

Figure S7 POM images of 5CB doped with $m$-TPE-NC on untreated glass slides.

Figure S8 AFM images of 5CB doped with $p$-TPE-OC at 2 wt.\% upon the evaporation of its THF solution on freshly cleaved mica substrates without annealing the sample.

Figure S9 AFM images of 5CB doped with $p$-TPE-NC at 2 wt.\% upon the evaporation of its THF solution on freshly cleaved mica substrates.

Figure S10 AFM images of 5CB doped with $p$-TPE-OC at 2 wt.\% upon the evaporation of its THF solution on freshly cleaved mica substrates.

Figure S11 AFM images of assemblies of $m$-TPE-NC and 5CB doped with $m$-TPE-NC at $2 \mathrm{wt} . \%$ upon the evaporation of their THF solution.

Figure S12 Illustration of modeled structures for $p$-TPE-NC assembly.

Figure S13 Plots of favored binding mode between 5CB molecules and $p$-TPE-NC assembly.

Figure S14 Illustration of modeled structures for $m$-TPE-OC assembly.

Figure S15 Plots of favored binding mode between 5CB molecules and $m$-TPE-OC assembly.

Figure S16 Plots of modeled fiber structures for TPE derivatives. 
Figure S17 ${ }^{1} \mathrm{H}$ NMR of compound $p$-TPE-NC.

Figure S18 ${ }^{13} \mathrm{C}$ NMR of compound $p$-TPE-NC.

Figure S19 HRMS of compound $p$-TPE-NC.

Figure S20 ${ }^{1} \mathrm{H}$ NMR of compound $m$-TPE-NC.

Figure S21 ${ }^{13} \mathrm{C}$ NMR of compound $m$-TPE-NC.

Figure S22 HRMS of compound $m$-TPE-NC.

Figure S23 ${ }^{1} \mathrm{H}$ NMR of compound $m$-TPE-OC.

Figure S24 ${ }^{13} \mathrm{C}$ NMR of compound $m$-TPE-OC.

Figure S25 HRMS of compound $m$-TPE-OC. 


\section{Materials and methods}

Pyridine, dimethylaminopyridine, cholesterol chloroformate were purchased from Energy Chemical Company. Solvents were purchased from Xilong Chemical Co., Ltd. All the chemicals were used as received without further purification. Compound 4-(1, 2, 2-triphenylvinyl) aniline ${ }^{1}, 3-\left(1,2,2\right.$-triphenylvinyl) aniline ${ }^{2}$ and 3-(1, 2, 2triphenylvinyl)phenol ${ }^{3}$ were prepared according to previous paper.

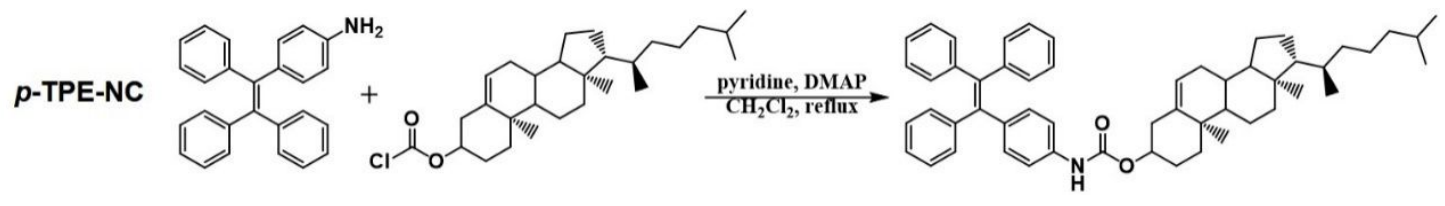

Scheme S1. Synthetic route of compound $p$-TPE-NC.

Synthesis of $\boldsymbol{p}$-TPE-NC: Compound 4-(1, 2, 2-triphenylvinyl) aniline (698 mg, 2 mmol), pyridine (0.39 mL, $2.5 \mathrm{mmol})$, dimethylaminopyridine (24 $\mathrm{mg})$ and cholesterol chloroformate $(978 \mathrm{mg}, 2.2 \mathrm{mmol})$ were dissolved in dry $\mathrm{CH}_{2} \mathrm{Cl}_{2}(20 \mathrm{~mL})$, and the resulting mixture was stirred and refluxed for $1 \mathrm{~d}$. After cooling, the reaction mixture was diluted with $\mathrm{CH}_{2} \mathrm{Cl}_{2}$, washed with water and brine, dried over $\mathrm{Na}_{2} \mathrm{SO}_{4}$. The solvents were removed under reduced pressure. The residue was purified by chromatography on a silica gel column (petroleum ether/dichloromethane $=1 / 2$ ) to yield the compound $p$ TPE-NC (1.3 g, 86.1\%) as white powder. ${ }^{1} \mathrm{H}$ NMR (400 MHz, $\left.\mathrm{CDCl}_{3}\right): \delta=7.12-7.08$ (m, 11H), 7.05-7.00 (m, 6H), $6.95(\mathrm{~d}, \mathrm{~J}=8.4 \mathrm{~Hz}, 2 \mathrm{H}), 6.43(\mathrm{~s}, 1 \mathrm{H}), 5.40-5.37(\mathrm{~m}, 1 \mathrm{H})$, 4.61-4.53 (m, 1H), 2.42-2.28 (m, 2H), 2.03-1.80 (m, 5H), 1.61-0.96 (m, 24H), $0.92(\mathrm{~d}$, $\mathrm{J}=6.4 \mathrm{~Hz}, 3 \mathrm{H}), 0.87(\mathrm{~d}, \mathrm{~J}=6.4 \mathrm{~Hz}, 6 \mathrm{H}), 0.68(\mathrm{~s}, 3 \mathrm{H}) \mathrm{ppm} ;{ }^{13} \mathrm{C}$ NMR $(100 \mathrm{MHz}$, $\left.\mathrm{CDCl}_{3}\right): \delta=153.0,144.0,143.9,140.7,140.5,139.7,138.8,136.5,132.2,131.5,131.5$, $127.9,127.8,127.8,126.6,126.5,126.5,122.9,122.9,117.7,75.0,56.8,56.3,50.1$, $42.5,39.9,39.7,38.6,37.1,36.7,36.3,36.0,32.1,32.0,28.4,28.2,28.2$, 24.4, 24.0, 23.0, 22.7, 21.2, 19.5, 18.9, 12.0 ppm; HRMS (ESI): $m / z$ calcd. for $\mathrm{C}_{54} \mathrm{H}_{65} \mathrm{NNaO}_{2}$ : $782.4913[M+\mathrm{Na}]^{+}$, found: 782.4908; element analysis calcd. (\%) for $\mathrm{C}_{54} \mathrm{H}_{65} \mathrm{NO}_{2}: \mathrm{C}$, 85.33; H, 8.62; N, 1.84; found: C, 84.88; H, 8.55; N, 1.8 


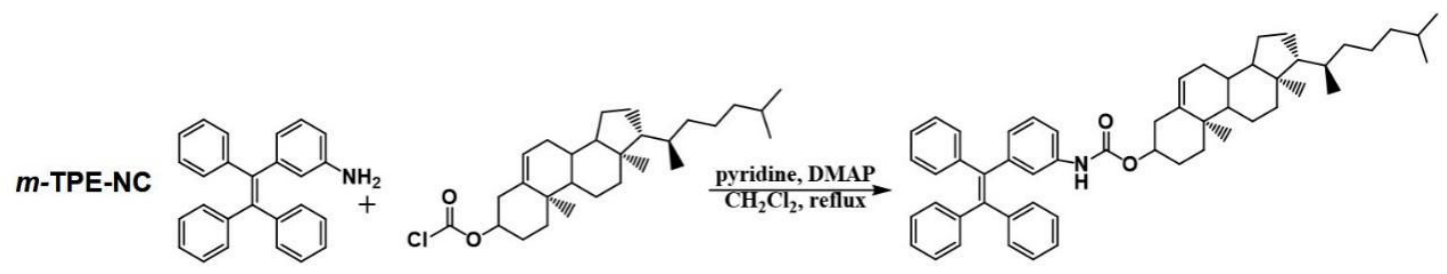

Scheme S2. Synthetic route of compound $m$-TPE-NC.

Synthesis of $\boldsymbol{m}$-TPE-NC: Compound 3-(1, 2, 2-triphenylvinyl) aniline (400 mg, 1.14 mmol), pyridine (0.22 mL, $2.5 \mathrm{mmol})$, dimethylaminopyridine (14 $\mathrm{mg})$ and cholesterol chloroformate $(565 \mathrm{mg}, 1.26 \mathrm{mmol})$ were dissolved in dry $\mathrm{CH}_{2} \mathrm{Cl}_{2}(20 \mathrm{~mL})$, and the resulting mixture was stirred and refluxed for $1 \mathrm{~d}$. After cooling, the reaction mixture was diluted with $\mathrm{CH}_{2} \mathrm{Cl}_{2}$, washed with water and brine, dried over $\mathrm{Na}_{2} \mathrm{SO}_{4}$. The solvents were removed under reduced pressure. The residue was purified by chromatography on a silica gel column (petroleum ether/dichloromethane $=1 / 2$ ) to yield the compound $m$ TPE-NC (750 mg, 86.0\%) as white powder. ${ }^{1} \mathrm{H}$ NMR (500 MHz, $\left.\mathrm{CDCl}_{3}\right): \delta=7.33$ (br, 1H), 7.13-7.07 (m, 9H), 7.05-7.00 (m, 7H), $6.83(\mathrm{t}, \mathrm{J}=1.7 \mathrm{~Hz}, 1 \mathrm{H}), 6.73(\mathrm{dt}, \mathrm{J}=7.7$, $1.0 \mathrm{~Hz}, 1 \mathrm{H}), 6.29(\mathrm{~s}, 1 \mathrm{H}), 5.39-5.36(\mathrm{~m}, 1 \mathrm{H}), 4.57-4.51(\mathrm{~m}, 1 \mathrm{H}), 2.38-2.24(\mathrm{~m}, 2 \mathrm{H})$, 2.03-1.80 (m, 5H), 1.60-1.00 (m, 24H), $0.92(\mathrm{~d}, \mathrm{~J}=6.5 \mathrm{~Hz}, 3 \mathrm{H}), 0.87(\mathrm{dd}, \mathrm{J}=6.7,2.3$ $\mathrm{Hz}, 6 \mathrm{H}), 0.68$ (s, 3H) ppm; $\left.{ }^{13} \mathrm{C} \mathrm{NMR} \mathrm{(126} \mathrm{MHz,} \mathrm{CDCl}_{3}\right): \delta=153.0,144.7,143.7$, $143.6,143.5,141.4,140.5,139.7,137.7,131.4,131.4,131.3,128.5,127.8,127.8,127.8$, $126.7,126.6,126.6,122.9,121.4,116.9,75.0,56.8,56.3,50.2,42.5,39.9,39.7,38.6$, 37.1, 36.7, 36.3, 36.0, 32.1, 32.0, 28.4, 28.2, 28.2, 24.4, 24.0, 23.0, 22.7, 21.2, 19.5, 18.9, 12.0 ppm; HRMS (ESI): $m / z$ calcd. for $\mathrm{C}_{54} \mathrm{H}_{65} \mathrm{NO}_{2}: 759.5015[M]^{+}$, found: 759.5009; element analysis calcd. (\%) for $\mathrm{C}_{54} \mathrm{H}_{65} \mathrm{NO}_{2}$ : $\mathrm{C}, 85.33 ; \mathrm{H}, 8.62 ; \mathrm{N}, 1.84$; found: C, 84.91; H, 8.41; N, 1.70 . 
$m$-TPE-OC
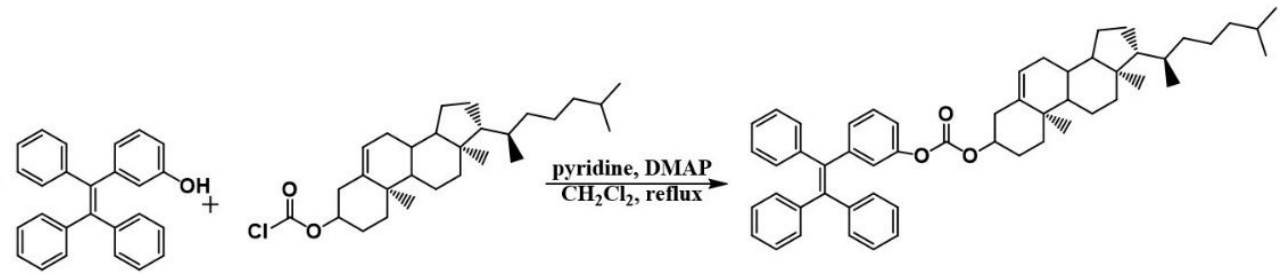

Scheme S3. Synthetic route of compound $m$-TPE-OC.

Synthesis of $\boldsymbol{m}$-TPE-OC: Compound 3-(1, 2, 2-triphenylvinyl) phenol (800 mg, 2.28 mmol), pyridine (0.45 $\mathrm{mL}, 5.7 \mathrm{mmol})$, dimethylaminopyridine $(27.9 \mathrm{mg})$ and cholesterol chloroformate $(1.13 \mathrm{mg}, 2.5 \mathrm{mmol})$ were dissolved in dry $\mathrm{CH}_{2} \mathrm{Cl}_{2}(20 \mathrm{~mL})$, and the resulting mixture was stirred and refluxed for $1 \mathrm{~d}$. After cooling, the reaction mixture was diluted with $\mathrm{CH}_{2} \mathrm{Cl}_{2}$, washed with water and brine, dried over $\mathrm{Na}_{2} \mathrm{SO}_{4}$. The solvents were removed under reduced pressure. The residue was purified by chromatography on a silica gel column (petroleum ether/dichloromethane $=2 / 1$ ) to yield the compound $m$-TPE-OC (1.54 g, 88.5\%) as white powder. ${ }^{1} \mathrm{H}$ NMR (500 MHz, $\left.\mathrm{CDCl}_{3}\right): \delta=7.13-7.07(\mathrm{~m}, 10 \mathrm{H}), 7.04-7.00(\mathrm{~m}, 6 \mathrm{H}), 6.95-6.93(\mathrm{~m}, 1 \mathrm{H}), 6.91-6.89(\mathrm{~m}$, $1 \mathrm{H}), 6.83(\mathrm{t}, \mathrm{J}=1.9 \mathrm{~Hz}, 1 \mathrm{H}), 5.41-5.38(\mathrm{~m}, 1 \mathrm{H}), 4.54-4.47(\mathrm{~m}, 1 \mathrm{H}), 2.46-2.36(\mathrm{~m}, 2 \mathrm{H})$, 2.03-1.95 (m, 3H), 1.91-1.80 (m, 2H), 1.71-1.02 (m, 24H), $0.92(\mathrm{~d}, \mathrm{~J}=6.5 \mathrm{~Hz}, 3 \mathrm{H})$, $0.87(\mathrm{dd}, \mathrm{J}=6.6,2.3 \mathrm{~Hz}, 6 \mathrm{H}), 0.68(\mathrm{~s}, 3 \mathrm{H}) \mathrm{ppm} ;{ }^{13} \mathrm{C} \mathrm{NMR}\left(126 \mathrm{MHz}, \mathrm{CDCl}_{3}\right): \delta=$ $152.9,150.9,145.4,143.6,143.4,143.3,141.9,139.9,139.3,131.5,131.4,131.4,129.0$, $128.6,127.9,127.9,127.8,126.8,126.7,126.7,124.0,123.3,119.3,78.8,56.8,56.3$, $50.1,42.5,39.9,39.7,38.1,37.0,36.7,36.3,35.9,32.1,32.0,28.4,28.2,27.8,24.4$, 24.0, 23.0, 22.7, 21.2, 19.4, 18.9, 12.0 ppm; HRMS (ESI): $m / z$ calcd. for $\mathrm{C}_{54} \mathrm{H}_{64} \mathrm{O}_{3}$ : $760.4855[M]^{+}$, found: 760.4850; element analysis calcd. (\%) for $\mathrm{C}_{54} \mathrm{H}_{64} \mathrm{O}_{3}: \mathrm{C}, 85.22$; H, 8.48; found: C, 85.04; H, 8.37.

\section{Theoretical calculation}

\section{Brief introduction for constructing helical structures of TPE derivatives fibers.}

In the fiber structure, we assumed that molecules are packed such that the intermolecular interactions are maximized. The inter-molecular interactions are influenced by two factors: the conformations of individual molecules and the packing modes 
between these molecules. Therefore, we first generated ensembles of representative conformations for each TPE derivative. For each conformation, we combined two monomers into dimers in which one monomer can perfectly overlap with the other one after $180^{\circ}$ rotation and translocation along the same axis. Using dimer as a unit, we further sampled fiber structures with different packing modes. Among these structures, the left-handed structure with highest inter-molecular interaction energy (or lowest inter-molecular potential energy) was selected as the modeled fiber structure.

\section{Generating representative conformations for TPE derivatives.}

The representative conformations of TPE derivatives were obtained after two steps of calculation: (1) performing molecular dynamic simulation (using GROMACS package ${ }^{4}$ with version 4.6.7) to sample different conformations and (2) selecting representative conformations. Here we use $m$-TPE-OC as an example to illustrate the detail procedures in two steps.

In molecular dynamics (MD) simulation, the force field of $m$-TPE-OC molecule was taken from gaff force field ${ }^{5}$ using antechamber. $m$-TPE-OC molecule was first solvated in a water box containing 1250 TIP3P water molecules. ${ }^{6}$ MD simulation was then performed under NPT ensemble ( 1.0 bar and $300 \mathrm{~K})$ with Berendsen pressure coupling 7 (coupling constant of $0.5 \mathrm{ps}^{-1}$ ) and velocity rescaling thermostat for temperature coupling $^{8}$ (coupling constant of $0.1 \mathrm{ps}^{-1}$ ). A cutoff radius of $10 \AA$ was used for van der Waals interaction. For coulomb interaction, Particle Mesh Ewald ${ }^{9}{ }^{10}$ was used to calculate the long-range electrostatic potential, and the radius for the short range was set at $10 \AA$. Neighbor lists were updated every 10 steps. All covalent bonds involving hydrogen atoms were constrained with LINCS. ${ }^{11}$ A 10000-step energy minimization was first performed using the steepest descent method. The solvent was then relaxed by applying a restrain potential on $m$-TPE-OC heavy atoms for $100 \mathrm{ps.} \mathrm{A} 400 \mathrm{~ns}$ simulation was performed for production. The configuration of $m$-TPE-OC molecule was saved every 2 ps, and 200,000 conformations in total were generated by MD simulation.

The 200,000 conformations of $m$-TPE-OC molecule were grouped into 300 clusters using k-center algorithm. In the clustering process, the distance between two 
conformations were measured by root mean square deviation (RMSD). After the clustering, 300 clusters of conformations were obtained, in which configurations from the same cluster show high structure similarity. Conformations corresponding to centers of the 300 clusters were selected as representative conformations.

For $p$-TPE-OC and $p$-TPE-NC, same calculation procedures were adopted except for the number of water molecules in box (1328 water molecules for $p$-TPE-OC and 1284 water molecules for $p$-TPE-NC).

\section{Combining two monomers into dimer.}

The crystal structure of $p$-TPE-OC (CCDC number 1887137) shows that two molecules are packed to form one packing unit with the symmetry that one molecule can overlap with the other one after $180^{\circ}$ rotation and translocation along the same axis. Based on this observation, we assumed that the packing unit in the helical fibers is composed of two TPE derivative monomers with similar organization. To obtain packed dimers with such symmetry, we first put two molecules of the same conformation apart and then optimize the relative positions between two molecules. The optimization process was performed by decreasing the inter-molecular van der Waals interaction using steepest decent algorithm (parameters for van der Waals interactions are taken from generalized AMBER force field ${ }^{2}$ ). In the optimization process, conformations of two molecules were fixed and the symmetry was maintained. For each representative conformation, we obtained 1000 packed dimer structures starting from different relative positions between two molecules before the optimization. Among the 1000 packing conformations, the identical conformations obtained from more than 20 different initial relative position were selected as favored dimer conformations, which were used to sample the structures of fibers in the next step. For $m$-TPE-OC, 1437 favored conformations were generated. This value was 1624 and 1587 for $p$-TPE-NC and $p$ TPE-OC, respectively.

\section{Modeling aggregate assemblies from aggregated dimers.}

Starting from one conformation of TPE derivative dimer as a unit, we replicated the 
dimer to build a dimer assembly consisting of 27 units $(3 * 3 * 3$ assembly). The 27 units are periodically organized in space. The relative positions of the 27 replicated units were then optimized by minimizing the potential energy of van der Waals interactions between these dimers (parameters for van der Waals interactions are taken from generalized AMBER force field). In the optimization process, the orientation and the periodic organization of the 27 dimer units were maintained. For each favored dimer conformation, 30,000 optimized assembly structures were generated starting from random initial assembly structures. For each TPE derivatives, more than 40 million of optimized assembly structures were generated, based on which the top 100 structures were selected according to the filtering of potential energy of van der Waals interactions. To further model the helical properties of each structure, the orientations of two neighbor dimers were relaxed with respect to the van der Waals interactions between them. After the relaxation, the orientations of neighbor dimers were shifted and a consecutive packed dimers according to the relaxed orientations lead to the helical profile. We finally filtered packing mode with left handed structures and then selected the one with the lowest potential energy.

\section{Simulate the interaction between $5 \mathrm{CB}$ molecules on the surface of the assemblies of TPE derivatives.}

To investigate the interactions between 5CB molecules and $m$-TPE-OC assemblies, we performed molecular dynamics simulation (using GROMACS package ${ }^{4}$ with version 4.6.7) to explore the possible binding modes of $5 \mathrm{CB}$ molecules on the surface of assemblies. In the system, the assembly was presented by $64 \mathrm{~m}$-TPE-OC molecules or 32 dimers (with the organization of $4 * 4 * 2$ ) with the conformations modeled by the method described above. The $64 \mathrm{~m}$-TPE-OC molecules were first located in the center of vacuum box $(9.7 \mathrm{~nm} * 9.2 \mathrm{~nm} * 10.5 \mathrm{~nm})$ with $105 \mathrm{CB}$ molecules randomly distributed in space. The simulation was performed under NVT ensemble (300 K) with velocity rescaling thermostat for temperature coupling ${ }^{5}$ (coupling constant of $0.1 \mathrm{ps}^{-1}$ ). A cutoff radius of $10 \AA$ was used for van der Waals interaction. For coulomb interaction, Particle Mesh Ewald ${ }^{9,10}$ was used to calculate the long-range electrostatic potential, and 
the radius for the short range was set at $10 \AA$. Neighbor lists were updated every 10 steps. All covalent bonds involving hydrogen atoms were constrained with LINCS. ${ }^{11} \mathrm{~A}$ 10000-step energy minimization was first performed using the steepest descent method. The structure of $m$-TPE-OC assembly was constrained by applying a restrain potential on all heavy atoms. A $1 \mathrm{~ns}$ simulation was performed and the final configuration was used for further analysis. Following these steps, 50 final configurations (500 configurations of $5 \mathrm{CB}$ molecules) were generated with each configuration simulated from different distribution of $5 \mathrm{CB}$ molecules in initial structure. The $5005 \mathrm{CB}$ configurations were statistically grouped into 20 clusters based on the structural similarity after the alignment of translocation. The configurations in the top cluster (with the highest number of configurations) were thought to corresponding to the favored binding mode. For $p$-TPE-NC and $p$-TPE-OC, similar procedures were performed with the difference in the sizes of simulation box $(10.3 \mathrm{~nm} * 10.4 \mathrm{~nm} * 8.9$ $\mathrm{nm}$ for $p$-TPE-NC and $8.0 \mathrm{~nm} * 11.3 \mathrm{~nm} * 7.9 \mathrm{~nm}$ for $p$-TPE-OC).

\section{REFERENCES}

(1) Luo, M.; Zhou, X.; Chi, Z.; Ma, C.; Zhang, Y.; Liu, S.; Xu. J. Synthesis and Properties of Gelators Derived from Tetraphenylethylene and Gallic Acid with Aggregation-Induced Emission. J Mol. Eng. Mater. 2013, 1, 1340007.

(2) Jiang, Y.; Wang, J; Huang, G.; Li Z.; Li B. S.; Tang, B. Z. Insight from the Old: Mechanochromism and Mechanoluminescence of Two Amine-Containing Tetraphenylethylene Isomers. J. Mater. Chem. C. 2019, 7, 11790-11796.

(3) Duan, X. F.; Zeng, J.; Lue, J. W.; Zhang, Z. B. A Facile Synthesis of Tetraarylethenes via Cross McMurry Coupling Between Diaryl Ketones. ChemInform. 2007, 5, 713-718.

(4) Hess, B.; Kutzner, C.; David, V. D.; Lindahl E. GROMACS 4: Algorithms for Highly Efficient, Load-Balanced, and Scalable Molecular Simulation. J. Chem. Theory Comput. 2008, 4, 435-447.

(5) Wang, J.; Wolf, R. M.; Caldwell, J. W.; Kollman, P. A.; Case, D. A. Development and Testing of a General Amber Force Field. J. Comput. Chem. 2004, 25, 1157-1174. 
(6) Jorgensen, W. L.; Chandrasekhar, J.; Madura, J. D.; Impey, R. W.; Klein, M. L. Comparison of Simple Potential Functions for Simulating Liquid Water. J. Chem. Phys. 1983, 79, 926-935.

(7) Berendsen, H. J. C.; Postma, J. P. M.; Vangunsteren, W. F.; Dinola, A.; Haak, J. R. Molecular Dynamics with Coupling to an External Bath. J. Chem. Phys. 1984, 81, 3684-3690.

(8) Bussi, G.; Donadio, D.; Parrinello, M. Canonical Sampling Through Velocity Rescaling. J. Chem. Phys. 2007, 126, 014101.

(9) Darden, T.; York, D.; Pedersen, L. Particle Mesh Ewald: An N · $\log (\mathrm{N})$ Method for Ewald Sums in Large Systems. J. Chem. Phys. 1993, 98, 10089-10092.

(10) Essmann, U.; Perera, L.; Berkowitz, M. A. Smooth Particle Mesh Ewald Method. J. Chem. Phys. 1995, 103, 8577-8593.

(11) Hess, B.; Bekker, H.; Berendsen, H. J. C.; Fraaije, J. G. LINCS: A Linear Constraint Solver for Molecular Simulations. J. Comput. Chem. 1997, 18, 1463-1472. 


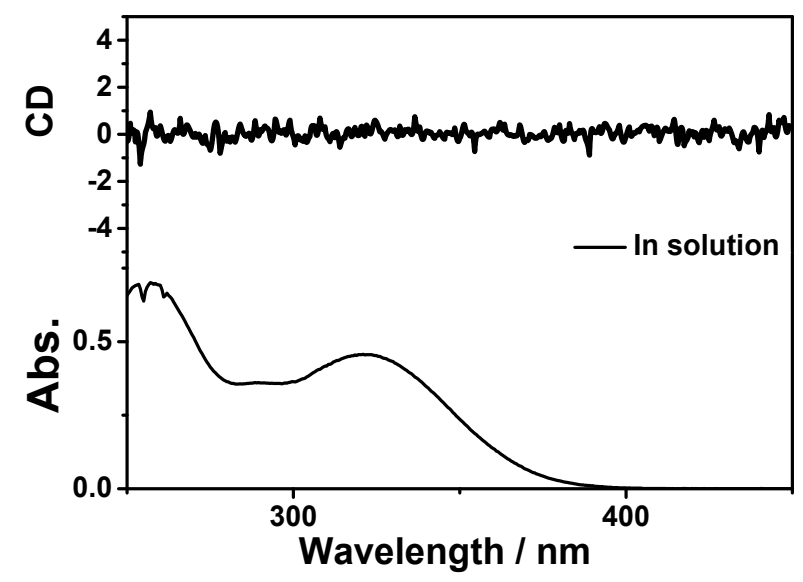

Figure S1. UV and CD spectra of $p$-TPE-NC in THF. Concentration: $10^{-4} \mathrm{M}$.
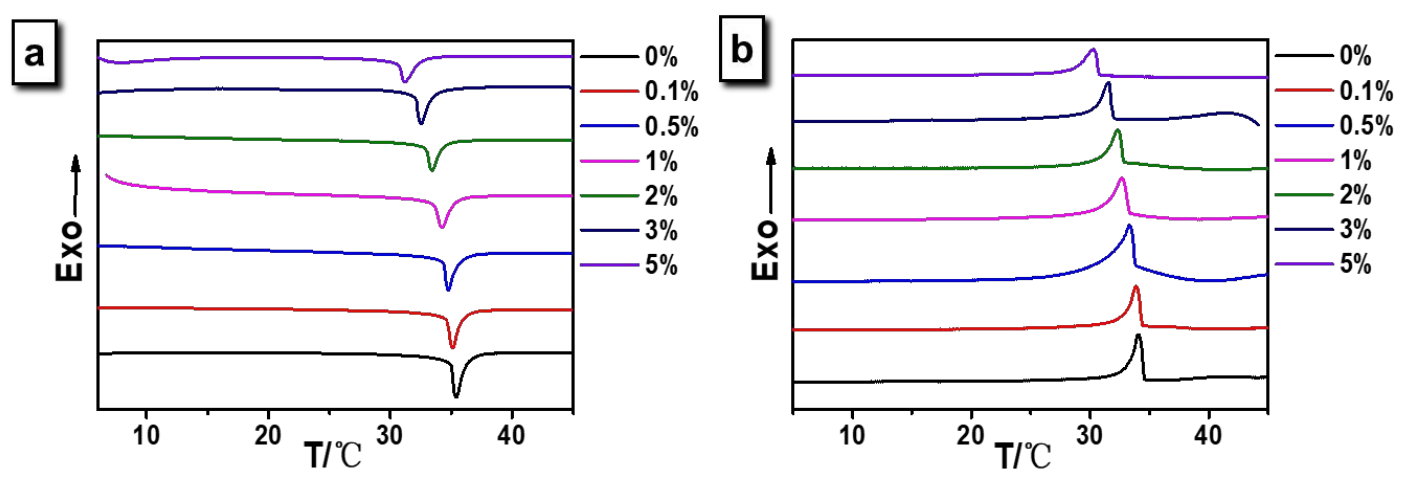

Figure S2. DSC plots of 5CB doped with $p$-TPE-NC at the ratios of $0.1-5$ wt.\%.

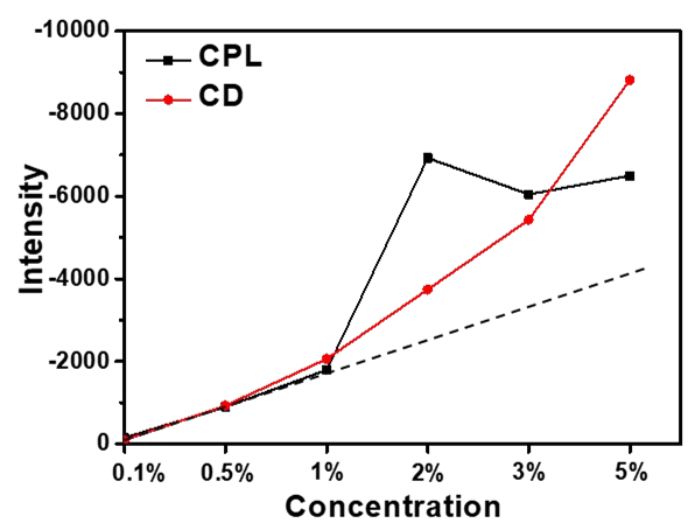

Figure S3. Plots of the concentration effect of $p$-TPE-NC on CD and CPL intensity in $5 \mathrm{CB}$. The dot line is provided for comparison with an ideal linear relationship. 

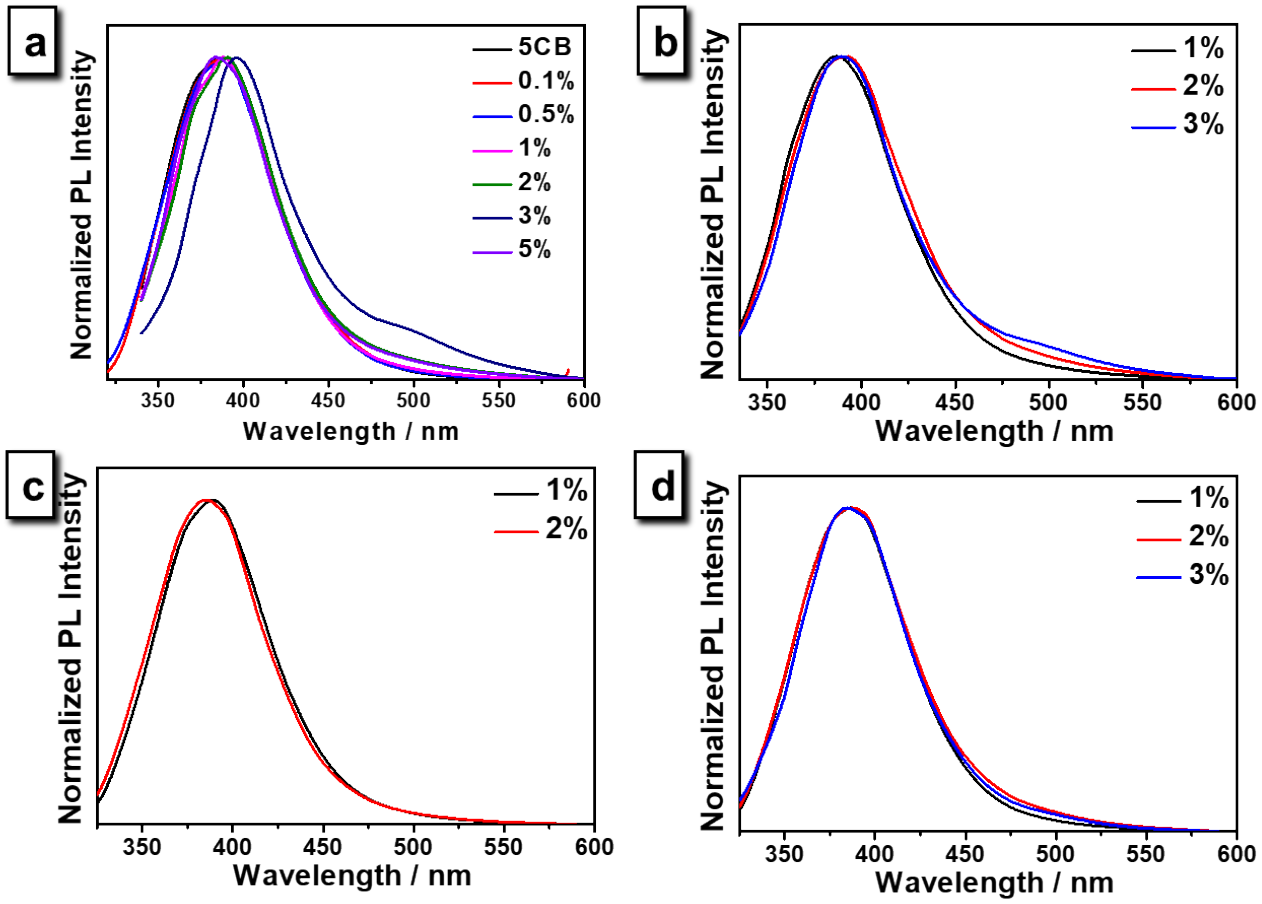

Figure S4. Fluorescence spectra of 5CB doped with (a) $0.1-5$ wt. $\% p$-TPE-NC, $\lambda_{\text {ex }}=$ $320 \mathrm{~nm}$, (b) $1-3$ wt. $\% p$-TPE-OC, $\lambda_{\text {ex }}=315 \mathrm{~nm}$, (c) $1-2$ wt. $\% m$-TPE-NC, $\lambda_{\text {ex }}=305$ nm, (d) 1 - 3 wt. $\% m$-TPE- OC, $\lambda_{\text {ex }}=305 \mathrm{~nm}$. 

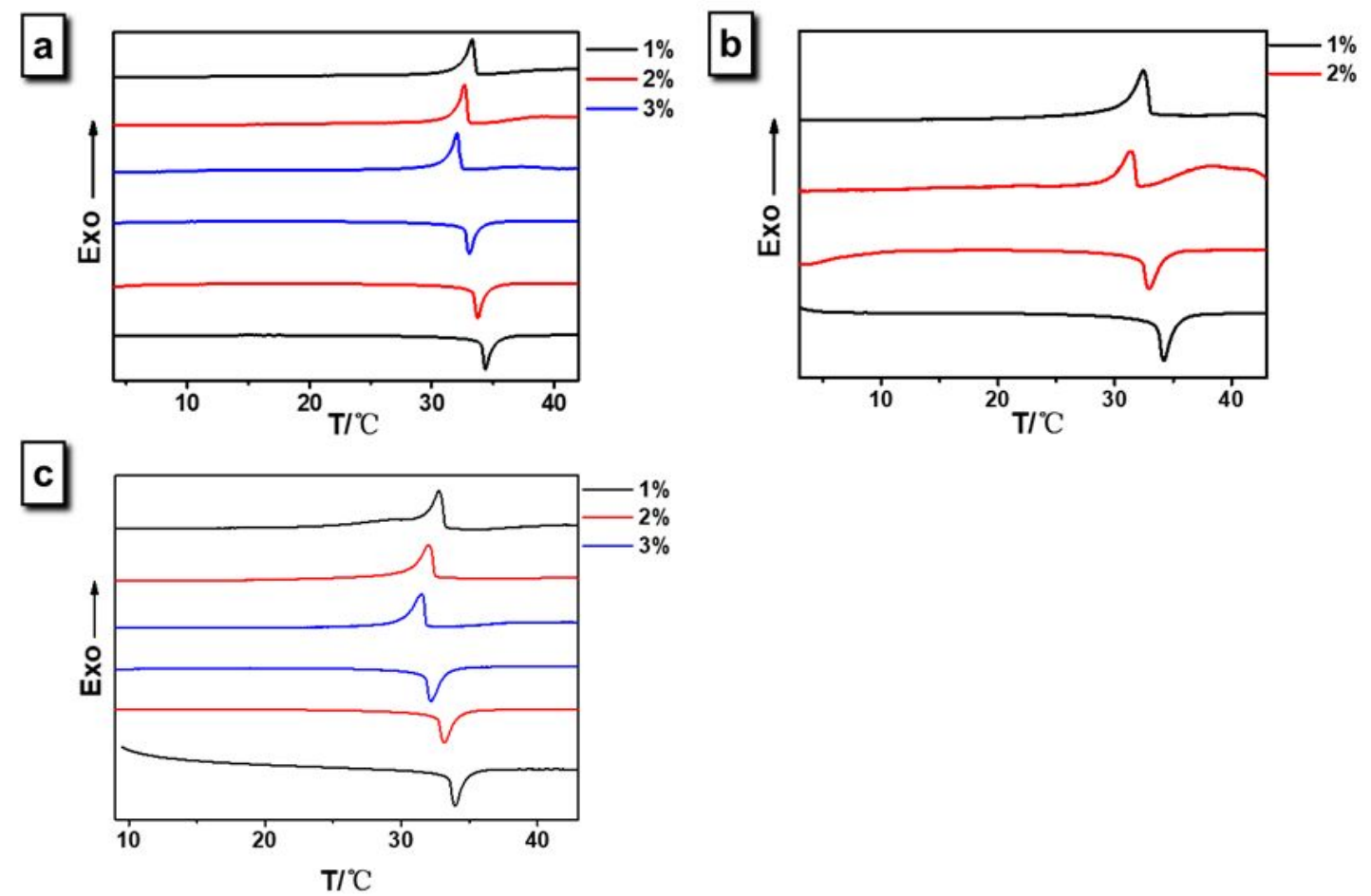

Figure S5. DSC plots of 5CB doped with (a) $p$-TPE-OC, (b) $m$-TPE-OC and (c) $m$ TPE-NC at a heating rate of $5 \mathrm{~K} \cdot \mathrm{min}^{-1}$ and a cooling rate of $5^{\circ} \mathrm{C} \cdot \mathrm{min}^{-1}$ in nitrogen. 

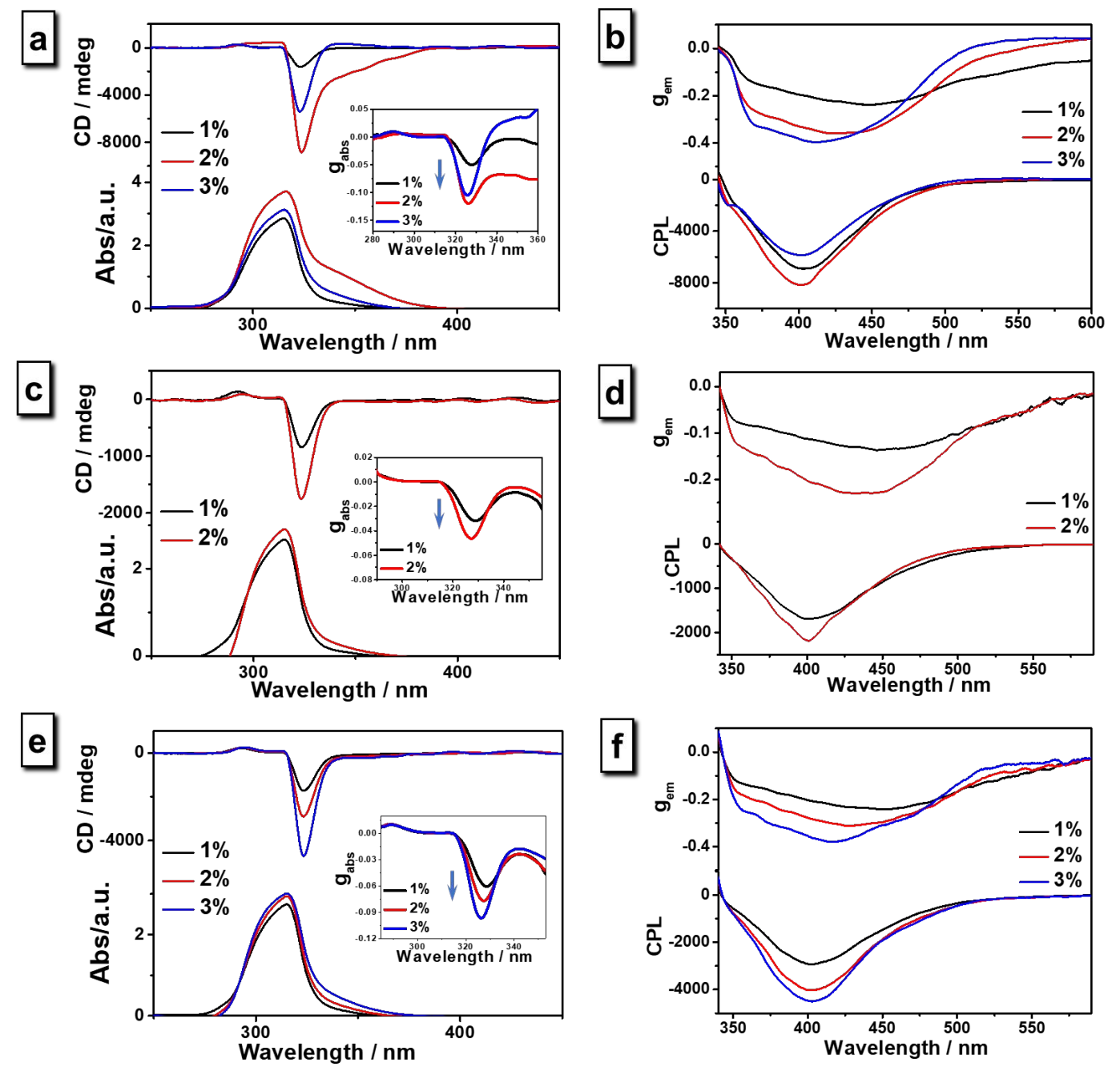

Figure S6. UV, CD and CPL spectra of 5CB doped with (a, b) $p$-TPE-OC, (c, d) $m$ TPE-NC and (e, f) $m$-TPE-OCn. $m$-TPE-OC, $\lambda_{\text {ex }}=315 \mathrm{~nm}$; $p$-TPE-OC, $m$-TPE-OC, $\lambda_{\text {ex }}$ $=305 \mathrm{~nm}$. Insert figures are $\mathrm{g}_{\mathrm{abs}}$ of corresponded CD spectra.

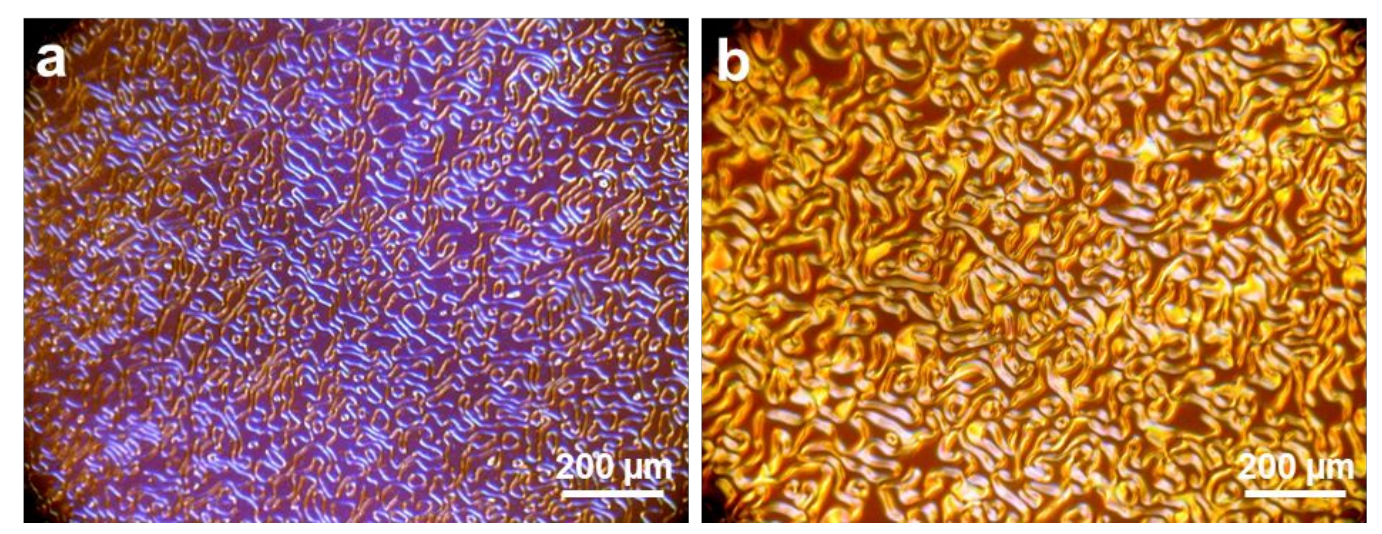

Figure S7. POM images of 5CB doped with $m$-TPE-NC on untreated glass slides at (a) 1 wt.\% and (b) 2 wt.\%. 


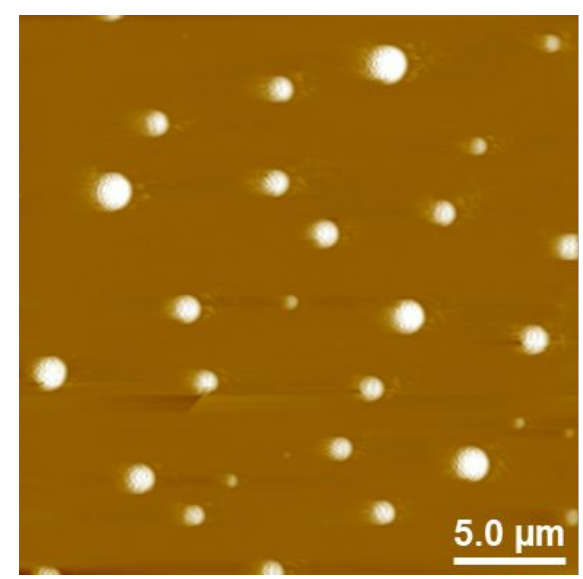

Figure S8. AFM images of 5CB doped with $p$-TPE-OC at $2 \mathrm{wt} \%$ upon the evaporation of its THF solution on freshly cleaved mica substrates without annealing the sample. Concentration: $0.029 \mathrm{mg} / \mathrm{ml}$.
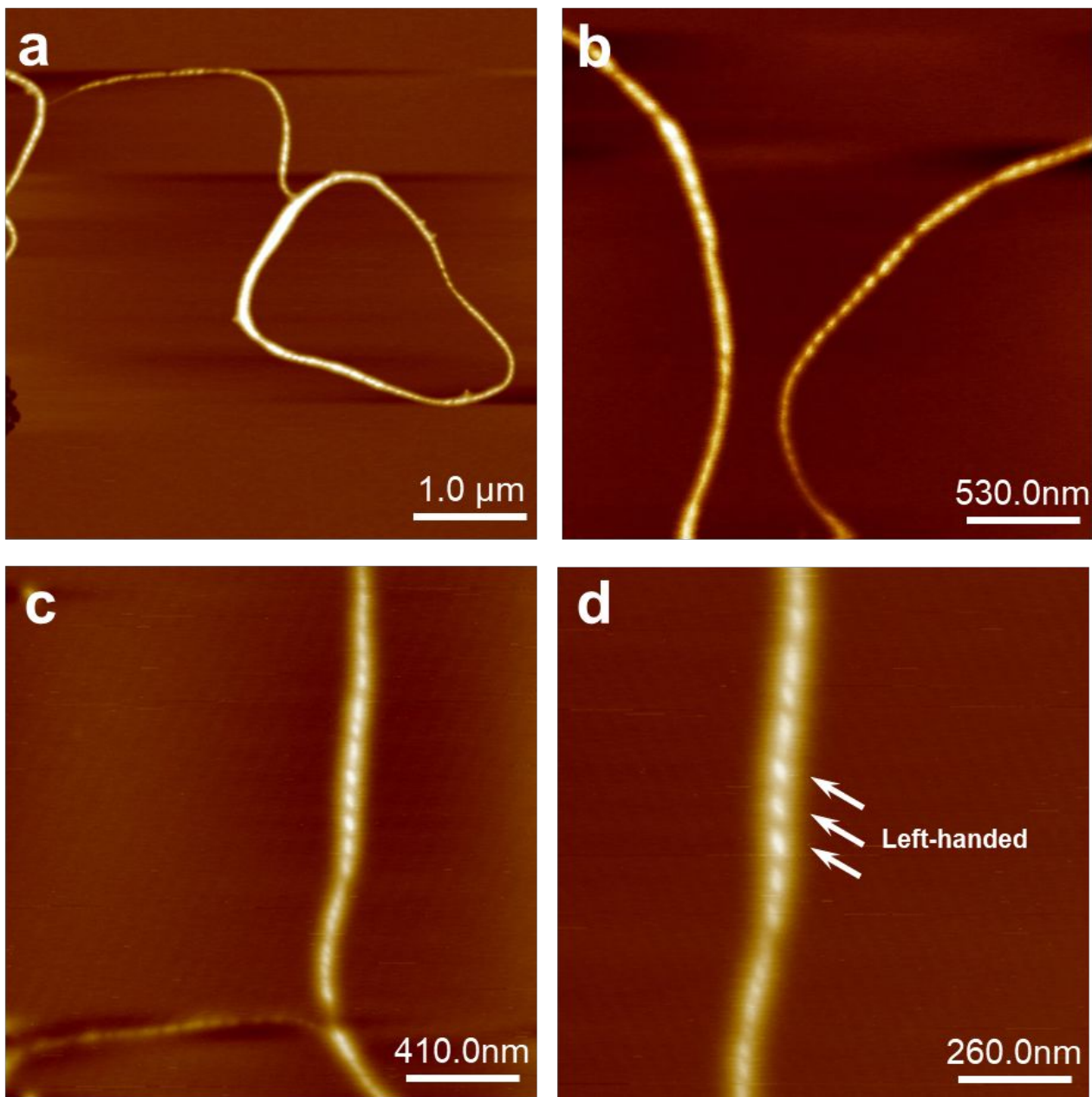

Figure S9. AFM images of 5CB doped with $p$-TPE-NC at 2 wt.\% upon the evaporation of its THF solution on freshly cleaved mica substrates. The samples were annealed for 
$72 \mathrm{~h}$ at $40{ }^{\circ} \mathrm{C}$ and slowly cooled to room temperature prior to AFM imaging. Arrows indicated the helical rotation of the beads.
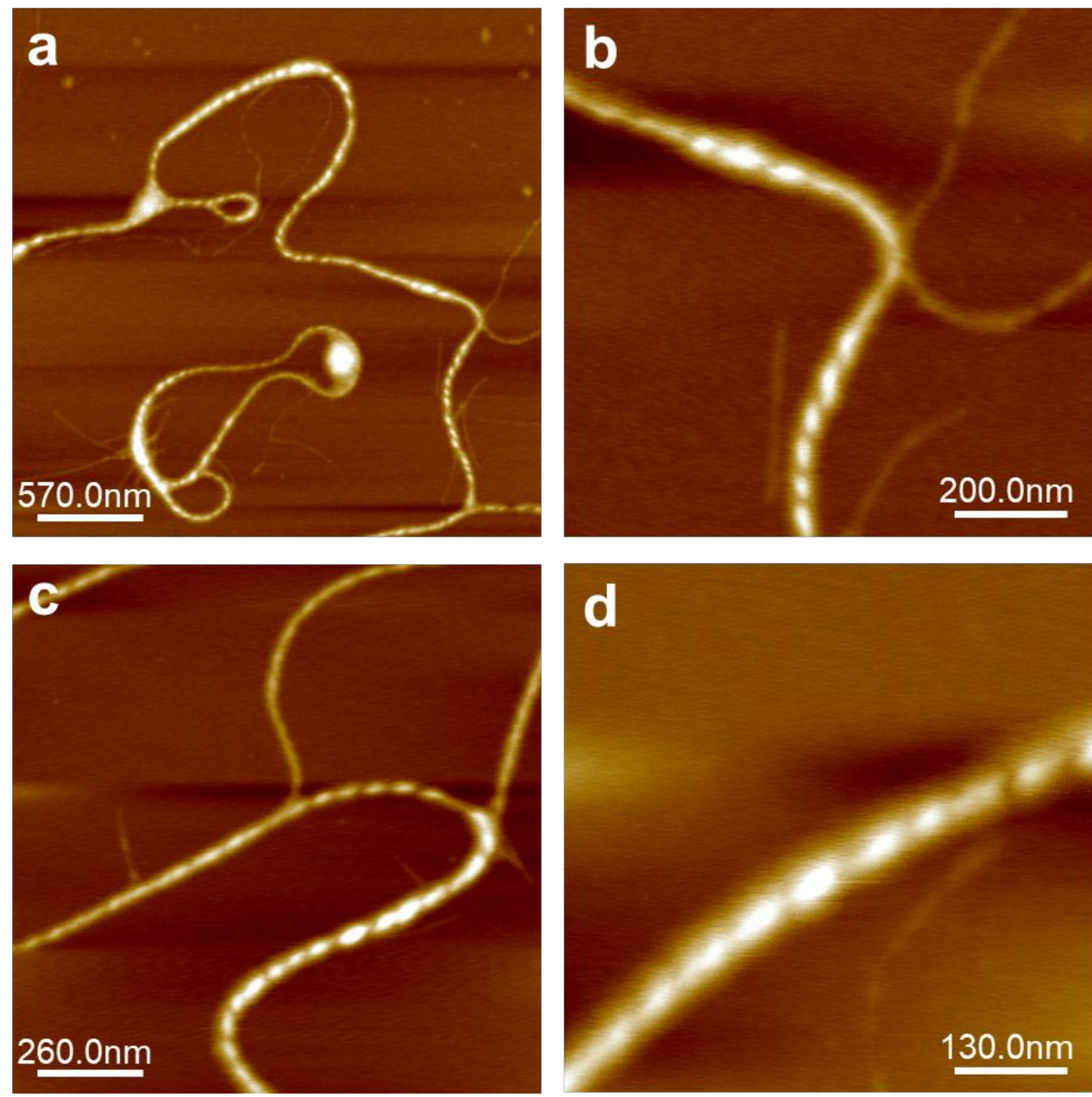

Figure S10. AFM images 5CB doped with $p$-TPE-OC at 2 wt.\% upon the evaporation of its THF solution on freshly cleaved mica substrates. The doped sample was annealed for $12 \mathrm{~h}$ at $40{ }^{\circ} \mathrm{C}$ and slowly cooled down to room temperature prior to AFM imaging. 


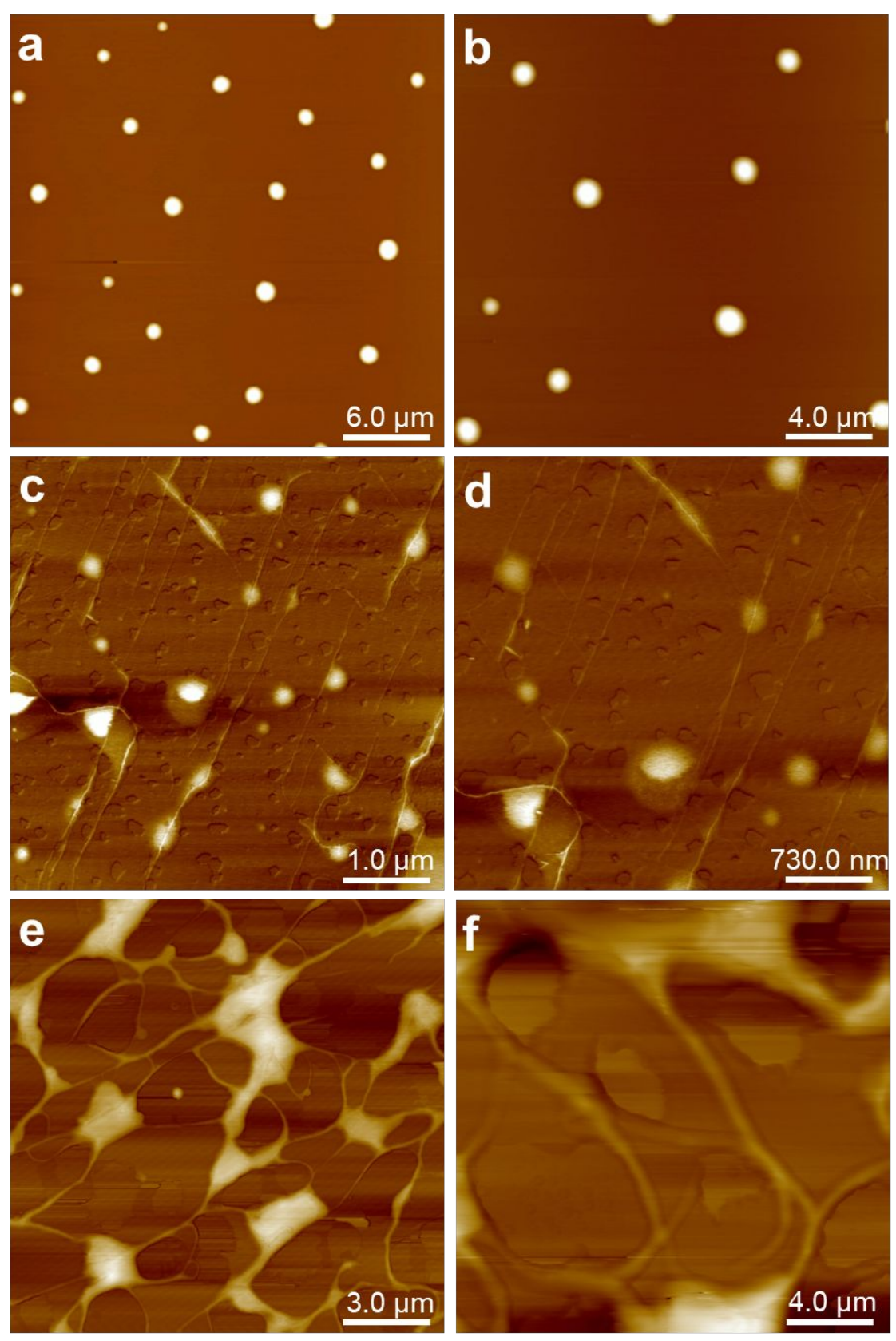

Figure S11. AFM images of assemblies of (a-d) $m$-TPE-NC and (e, f) 5CB doped with $m$-TPE-NC at 2 wt.\% upon the evaporation of their THF solution on freshly cleaved mica substrates. The doped sample was annealed for $12 \mathrm{~h}$ at $40{ }^{\circ} \mathrm{C}$ and slowly cooled down to room temperature prior to imaging. 
a
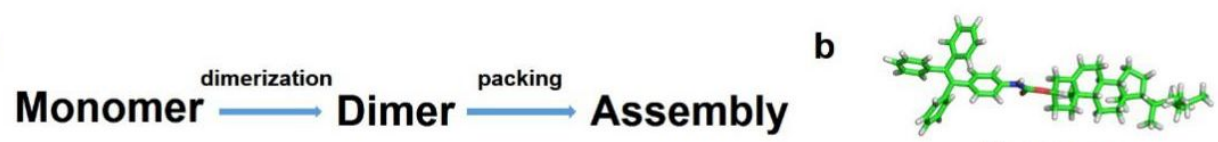

Monomer

C
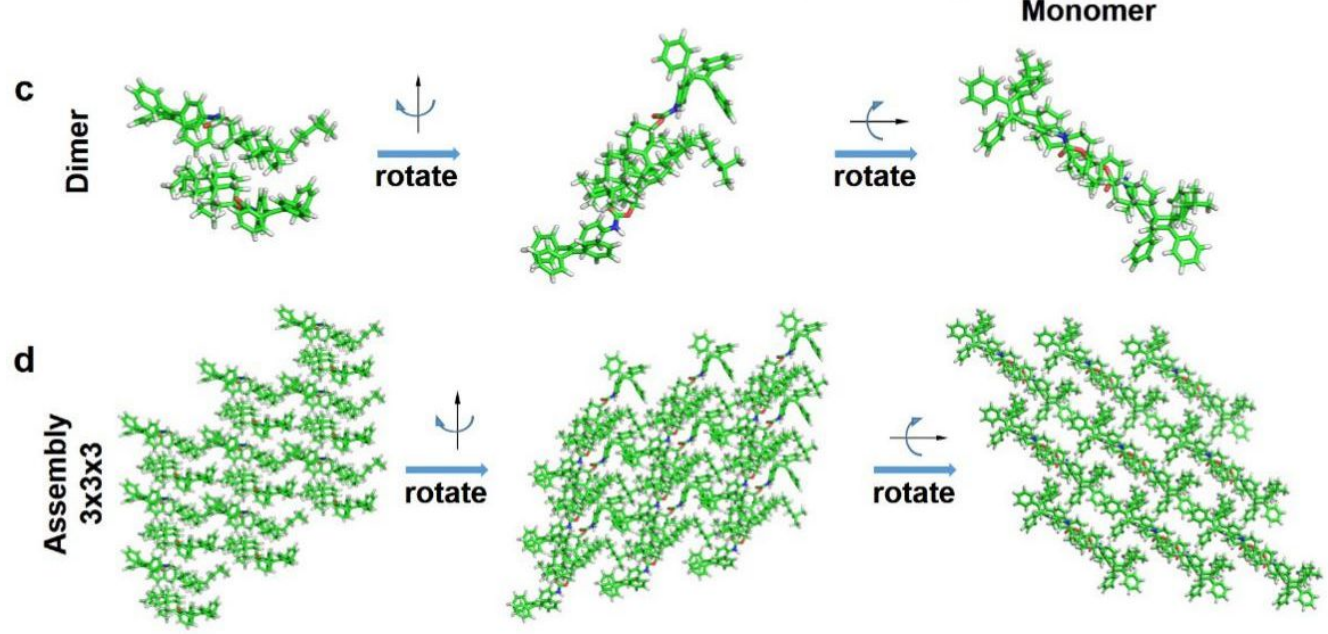

Figure S12. Illustration of modeled structures for $p$-TPE-NC assembly. Diagram plot of (a) the hierarchical organization of molecules and (b) the conformation of $p$-TPE$\mathrm{NC}$ monomer in modeled assembly. 3D visualization of (c) modeled dimer and (d) an assembly segment consisting of $3 * 3 * 3$ dimers from different views. In this plot, molecules are shown with stick representation, ie chemical bonds are shown as sticks. Atoms in molecules are labeled with different colors: white for hydrogen, green for carbon, red for oxygen. 


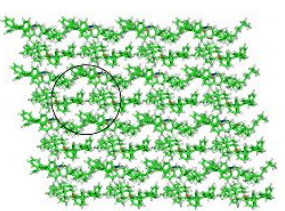

b

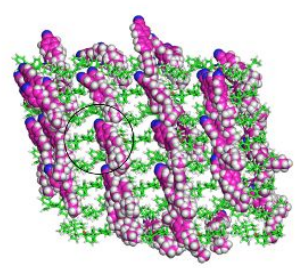

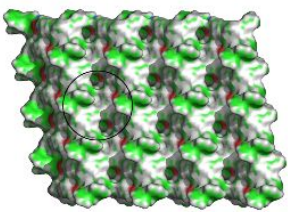

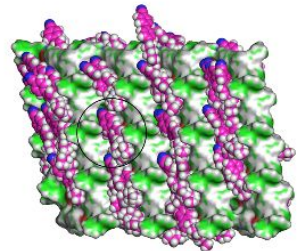

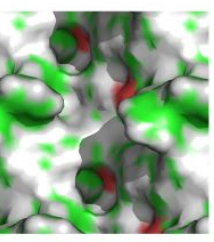

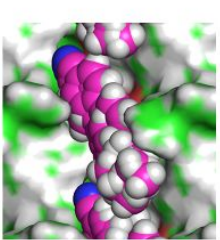

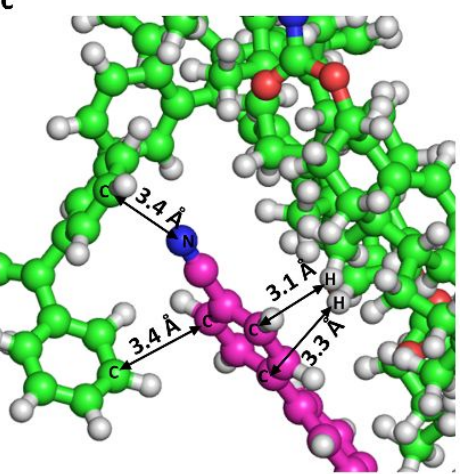

Figure S13. Plots of favored binding mode between 5CB molecules and $p$-TPE-NC assembly. (a) The structure of assembly consisting of $4 * 4 * 2$ dimers are shown with stick representation (left), surface representation (middle) and zoomed view for the confined space marked by black circle (right). (b) Plot of the favored binding mode of 5CB molecules on $p$-TPE-NC assembly. Conformations of 5CB molecules (colored in magenta) are superimposed in this plot with atoms shown as spheres. The assembly is plotted with the same representation shown in (a). (c) Structural analysis of the intermolecular interactions between 5CB and neighboring $p$-TPE-NC molecules shown in the right panel in (b). Atoms in molecules are labeled with different colors: white for hydrogen, green for carbon in $p$-TPE-NC, magenta for carbon in $5 \mathrm{CB}$, red for oxygen, blue for nitrogen. 
a

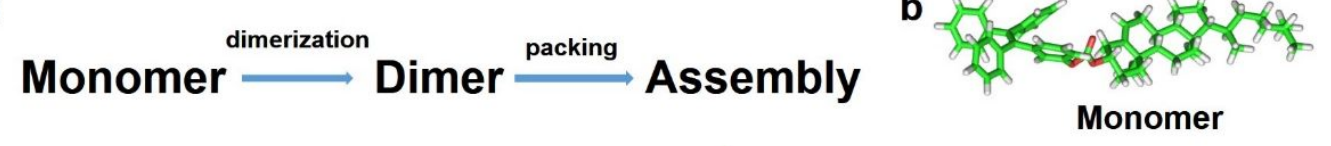

C
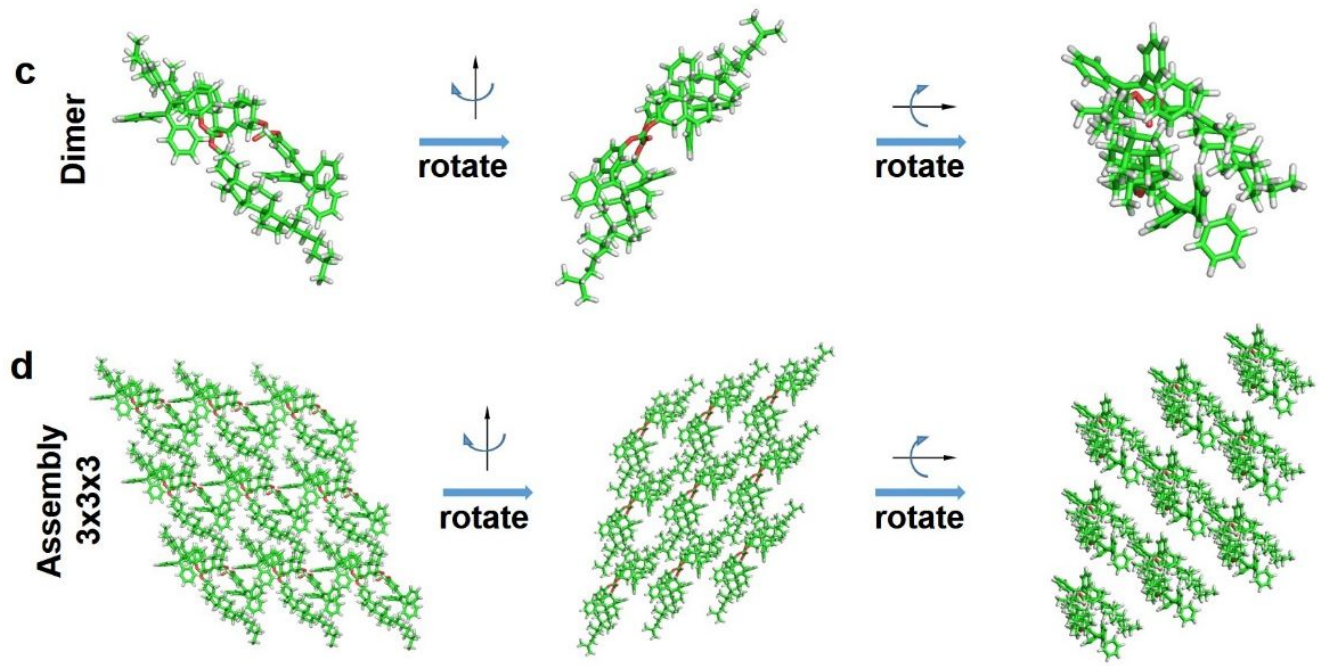

Figure S14. Illustration of modeled structures for $m$-TPE-OC assembly. Diagram plot of (a) the hierarchical organization of molecules and (b) the conformation of $m$-TPEOC monomer in modeled assembly. 3D visualization of (c) modeled dimer and (d) an assembly segment consisting of $3 * 3 * 3$ dimers from different views. In this plot, molecules are shown with stick representation, ie chemical bonds are shown as sticks. Atoms in molecules are labeled with different colors: white for hydrogen, green for carbon, red for oxygen. 
a

b

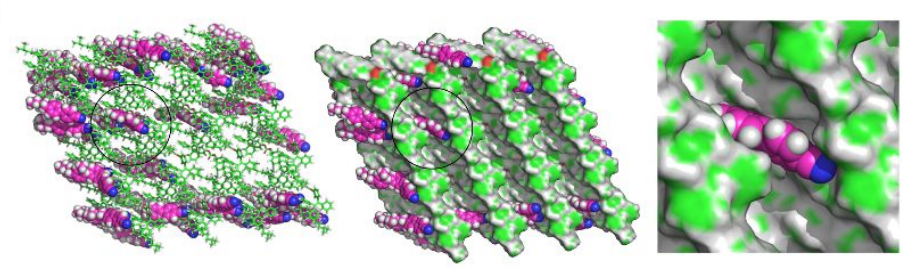

c

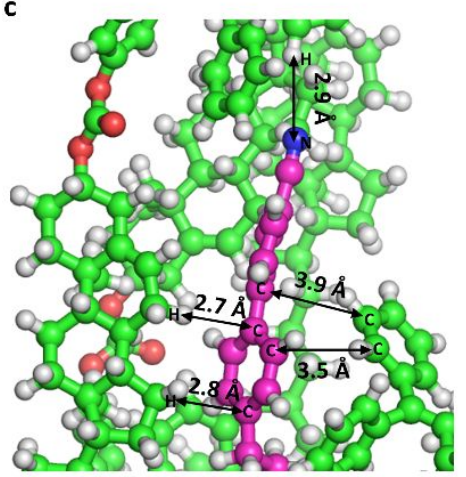

Figure S15. Plots of favored binding mode between 5CB molecules and $m$-TPE-OC assembly. (a) The structure of assembly consisting of $4 * 4 * 2$ dimers are shown with stick representation (left), surface representation (middle) and zoomed view for the confined space marked by black circle (right). (b) Plot of the favored binding mode of 5CB molecules on $m$-TPE-OC assembly. Conformations of 5CB molecules (colored in magenta) are superimposed in this plot with atoms shown as spheres. The assembly is plotted with the same representation shown in (a). (c) Structural analysis of the intermolecular interactions between $5 \mathrm{CB}$ and neighboring $m$-TPE-OC molecules shown in the right panel in (b). Atoms in molecules are labeled with different colors: white for hydrogen, green for carbon in $m$-TPE-OC, magenta for carbon in 5CB, red for oxygen, blue for nitrogen.

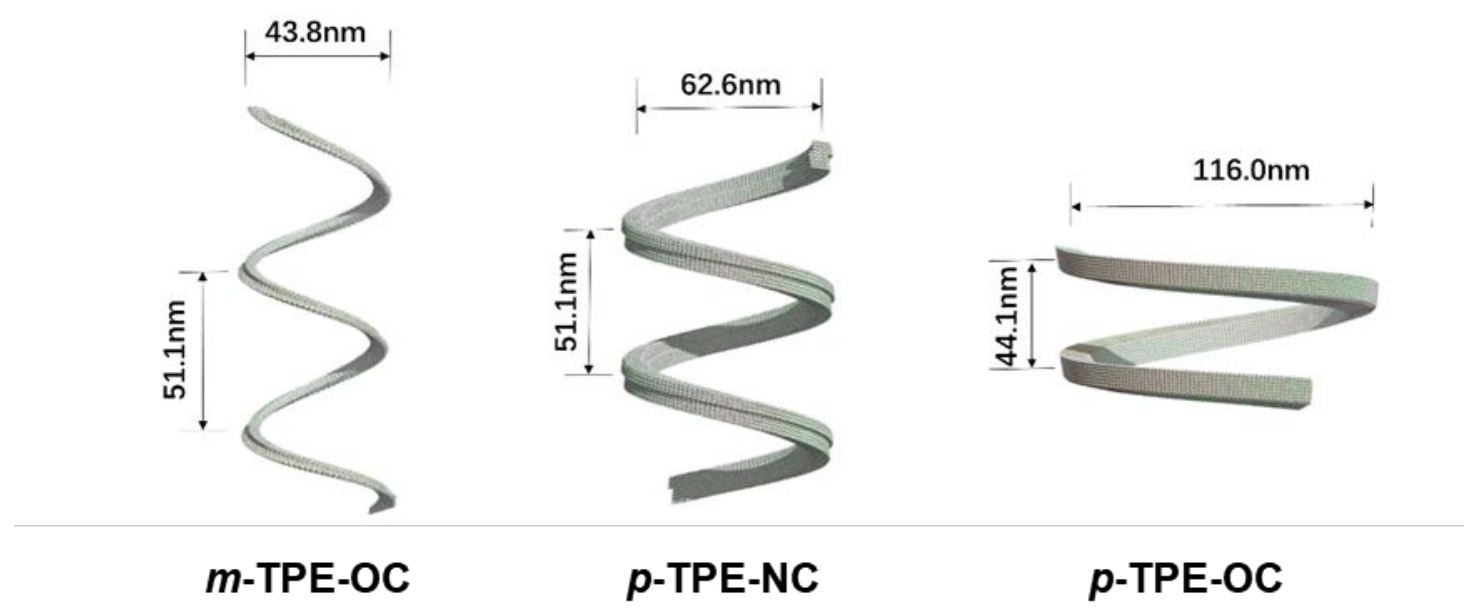

Figure S16. Plots of modeled fiber structures for TPE derivatives. 


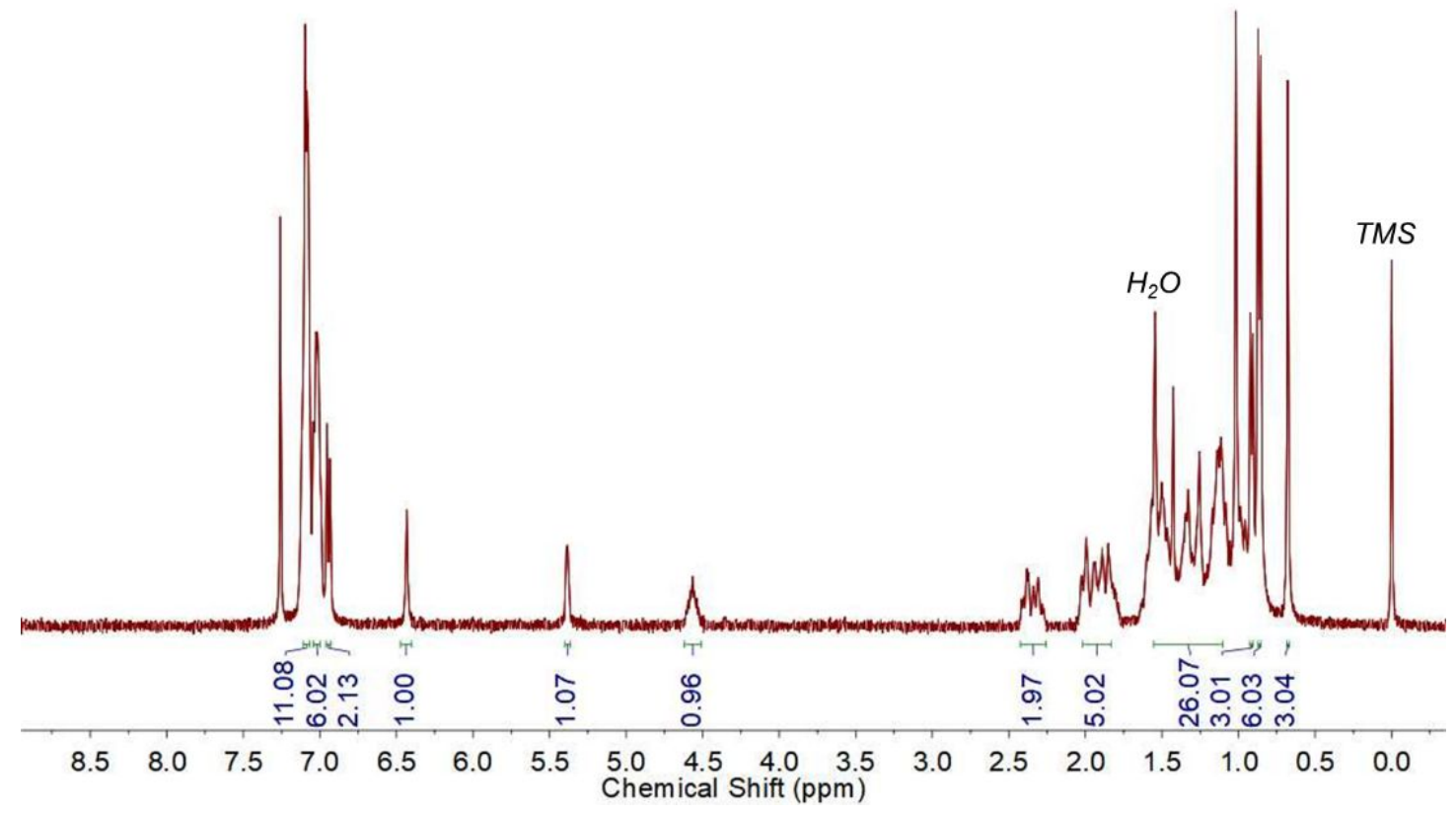

Figure S17. ${ }^{1} \mathrm{H}$ NMR of compound $p$-TPE-NC.

๑

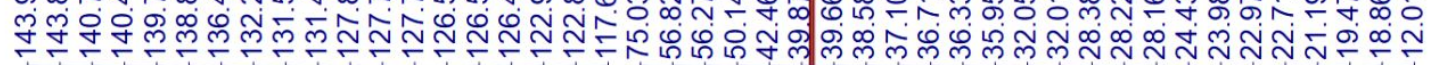

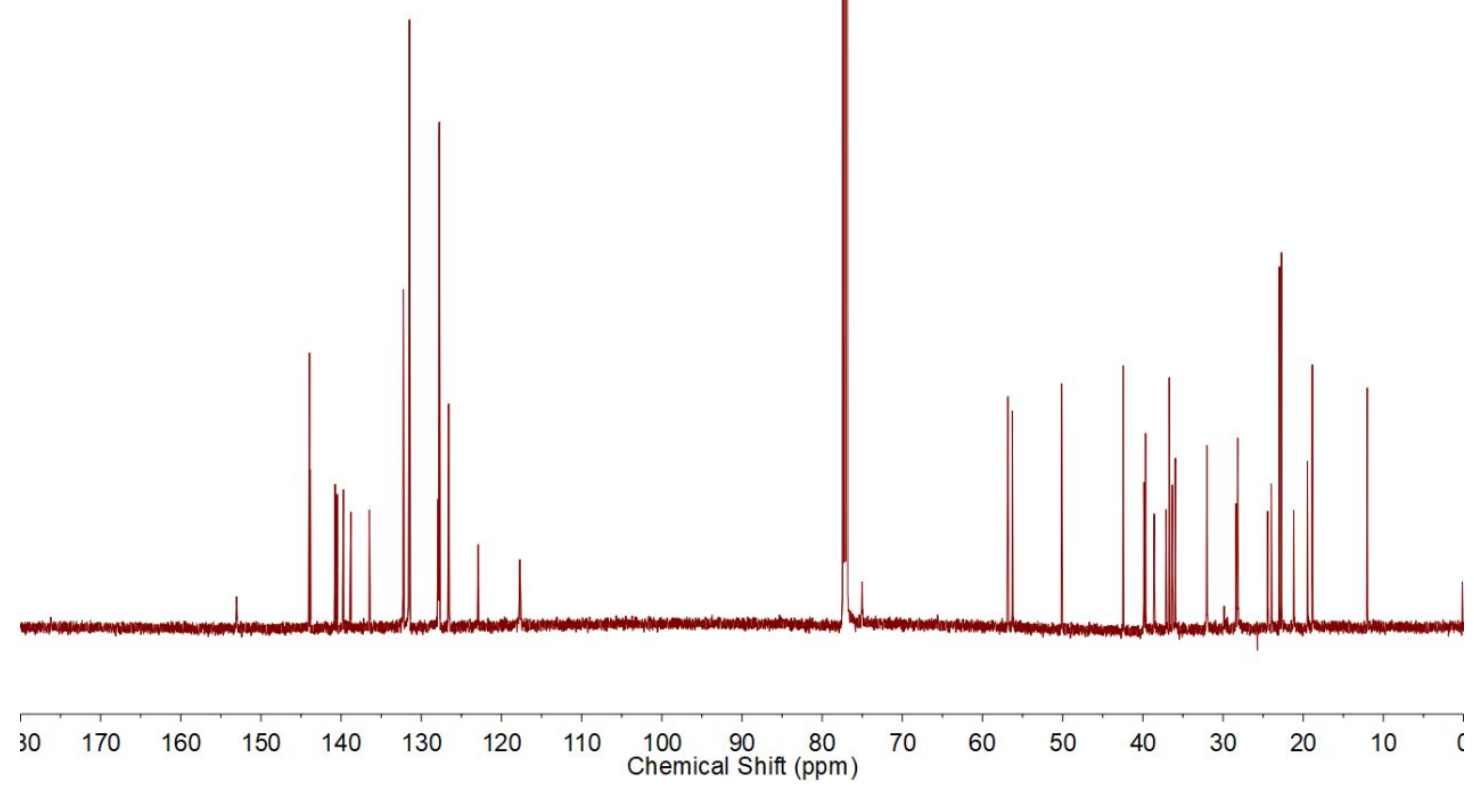

Figure S18. ${ }^{13} \mathrm{C}$ NMR of compound $p$-TPE-NC. 


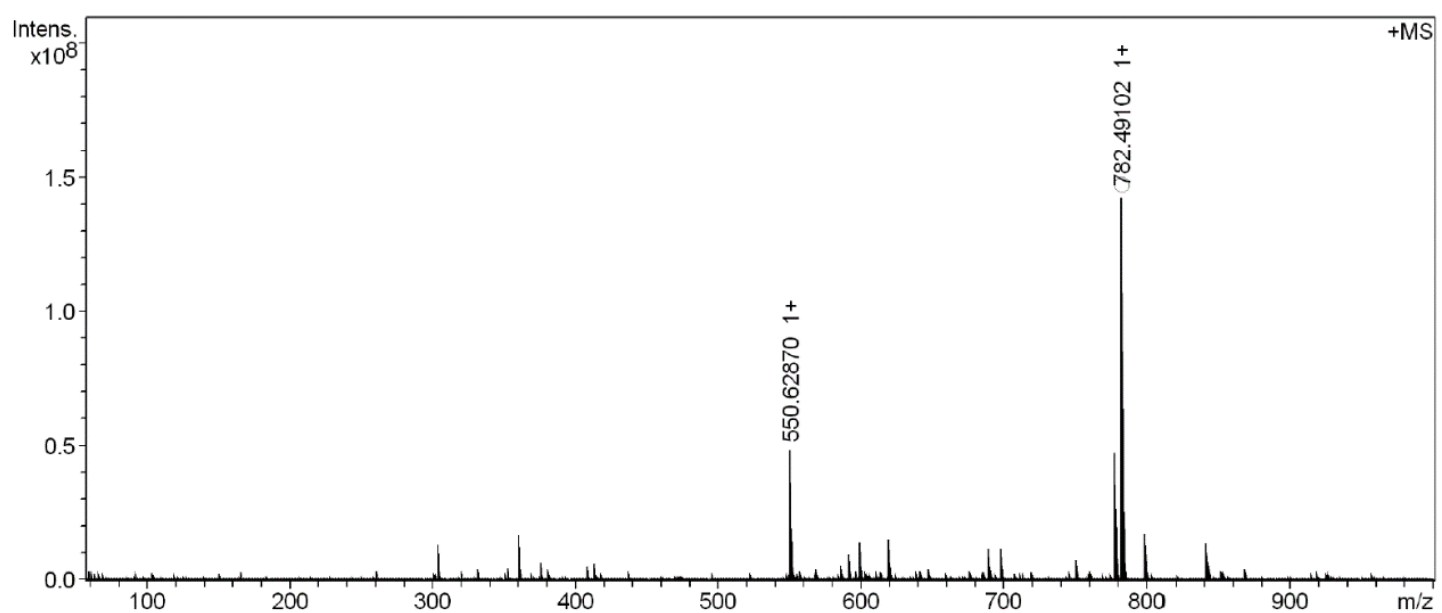

Figure S19. HRMS of compound $p$-TPE-NC.

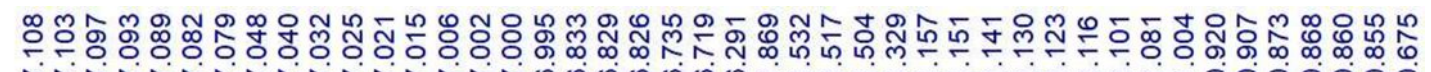

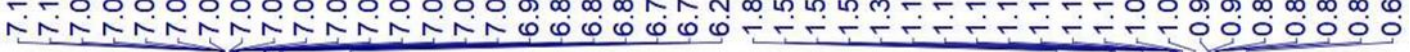

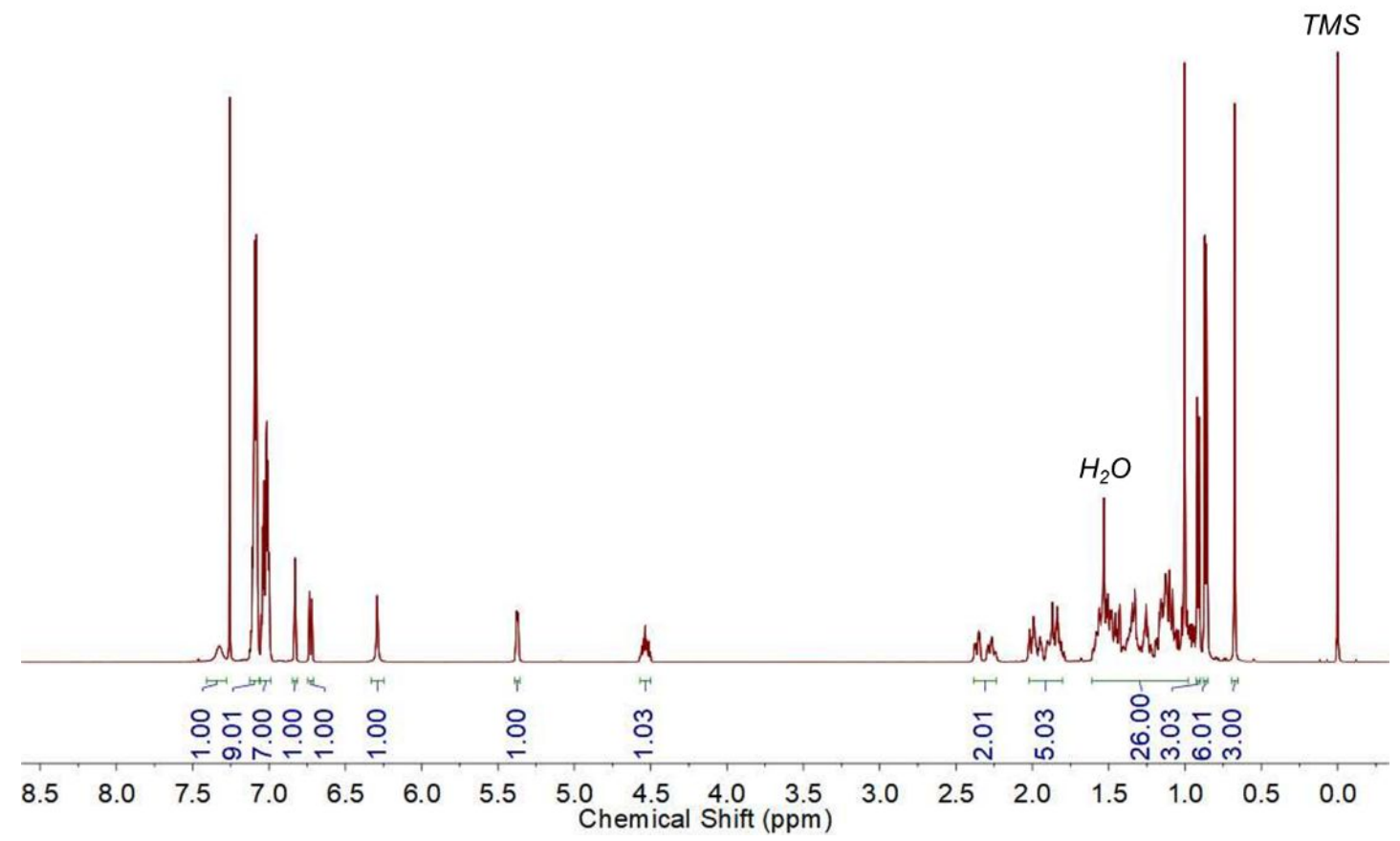

Figure S20. ${ }^{1} \mathrm{H}$ NMR of compound $m$-TPE-NC. 


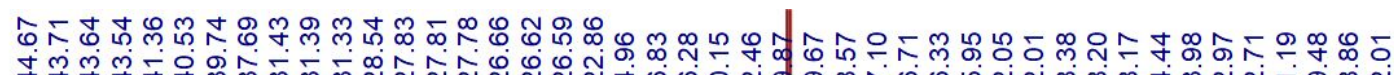

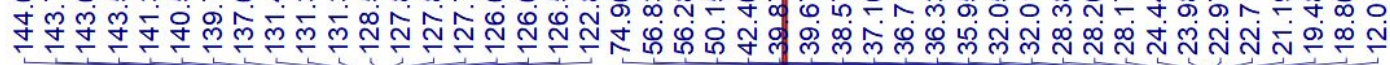

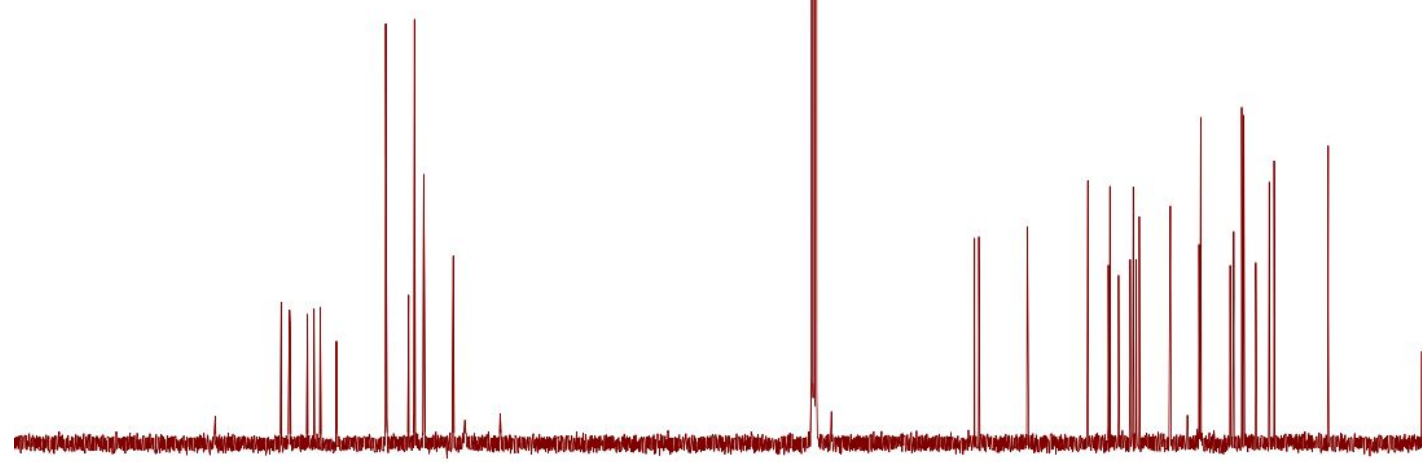

$\begin{array}{lllllllllllllll}170 & 160 & 150 & 140 & 130 & 120 & 110 & \begin{array}{c}100 \\ \text { Chemical Shift (ppm) }\end{array} & \begin{array}{l}80 \\ \text { Chem }\end{array} & 60 & 50 & 40 & 30 & 20 & 10\end{array}$

Figure S21. ${ }^{13} \mathrm{C}$ NMR of compound $m$-TPE-NC.

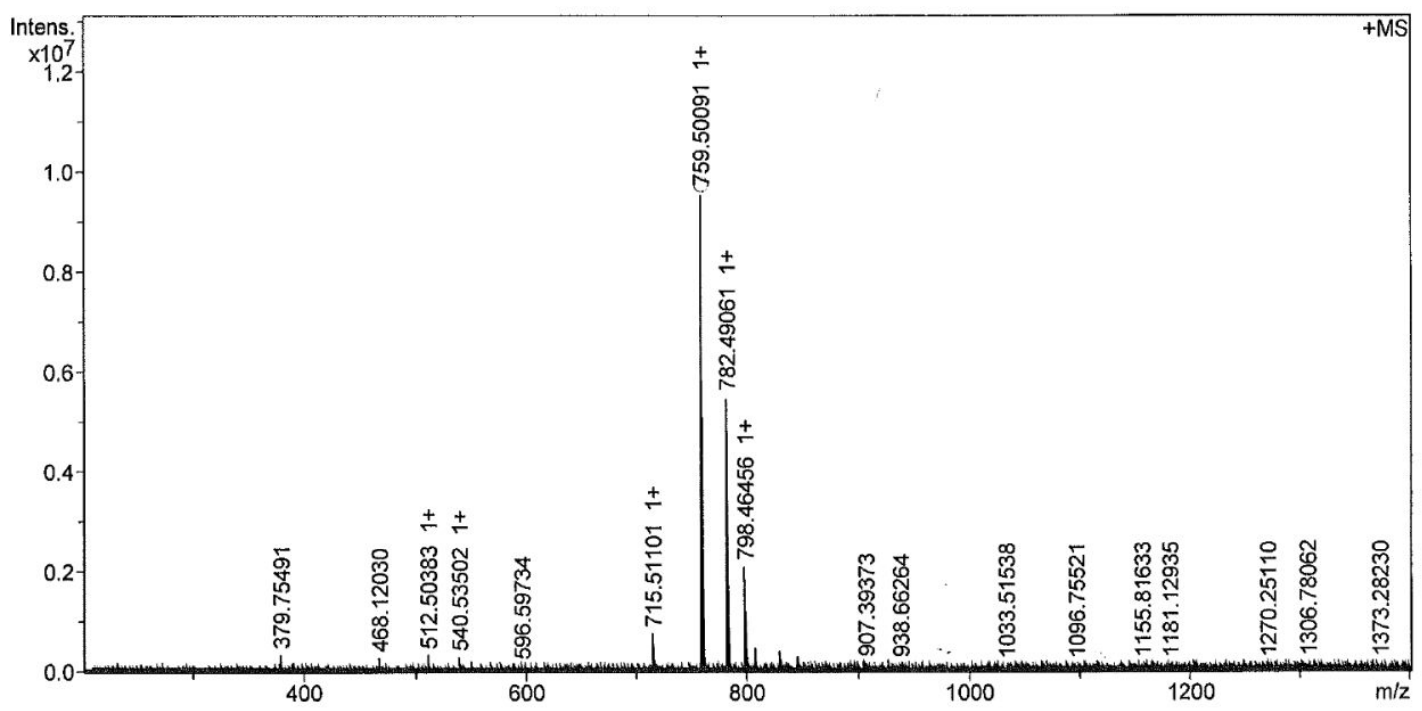

Figure S22. HRMS of compound $m$-TPE-NC. 


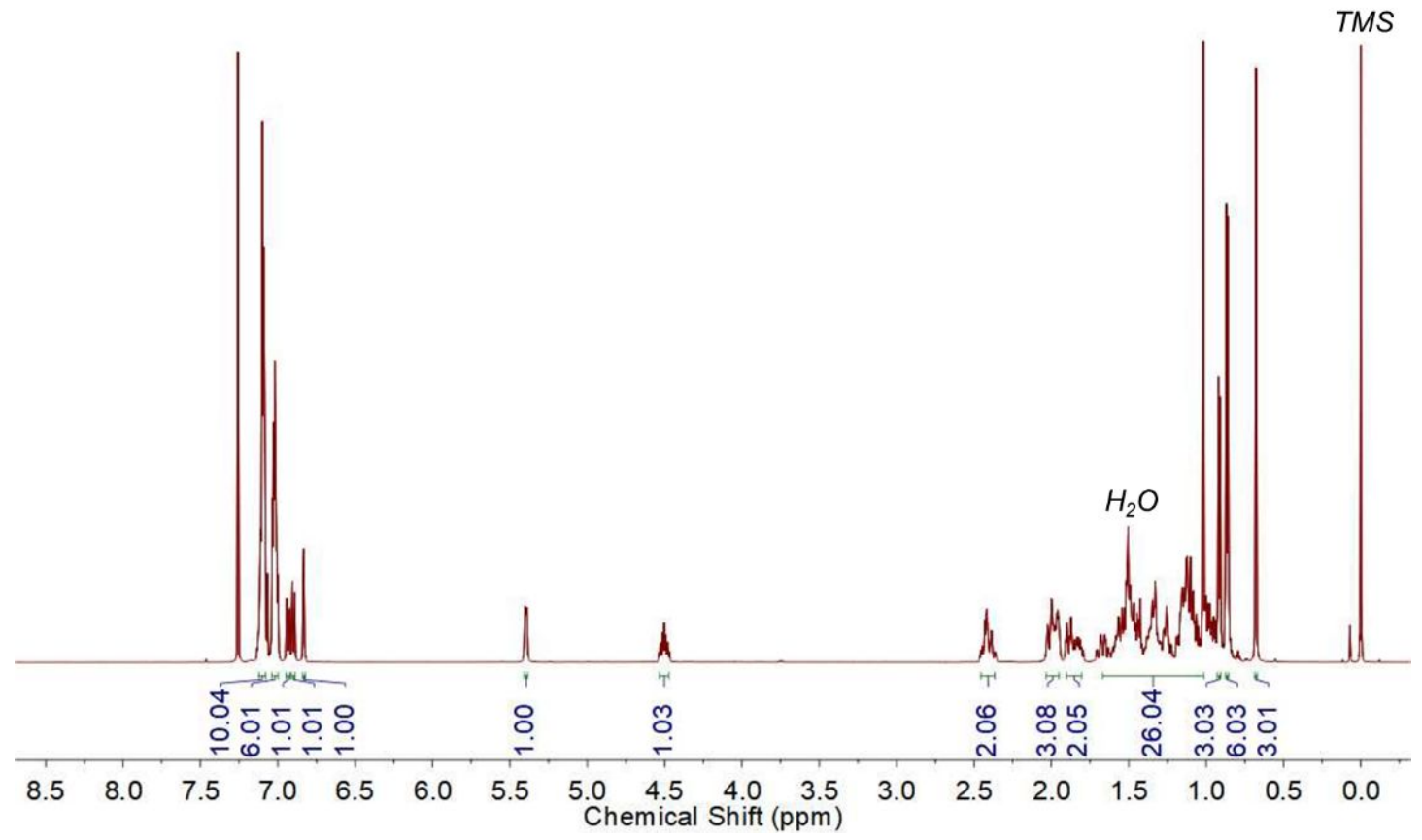

Figure S23. ${ }^{1} \mathrm{H}$ NMR of compound $m$-TPE-OC.

œ

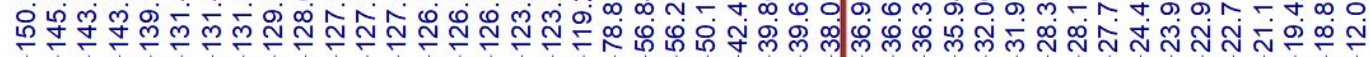

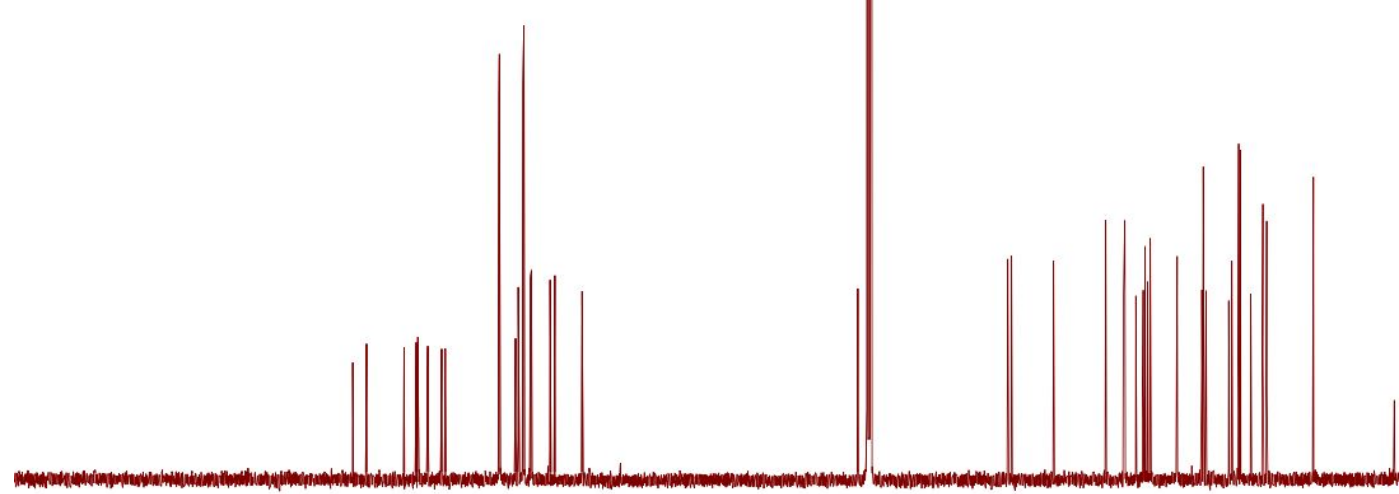

$190 \quad 170 \quad 150 \quad 130$ Chemical Shift (ppm)

Figure S24. ${ }^{13} \mathrm{C}$ NMR of compound $m$-TPE-OC. 


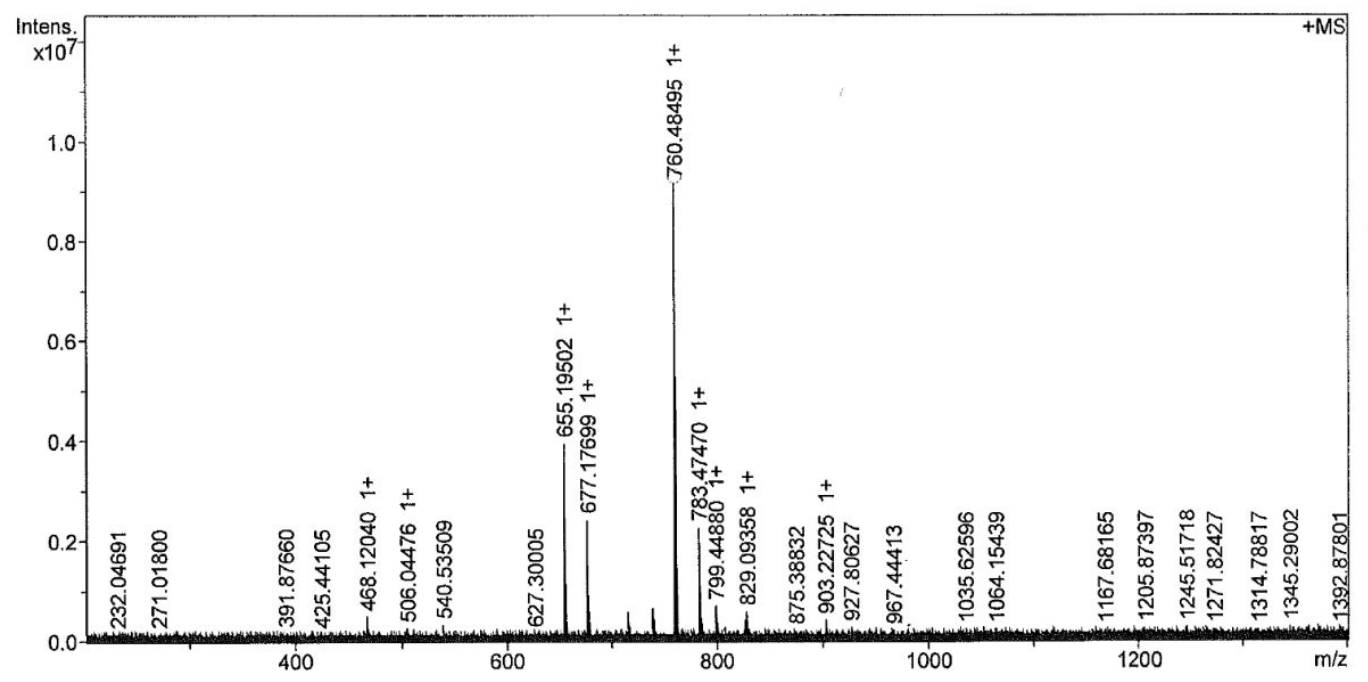

Figure S25. HRMS of compound $m$-TPE-OC. 